\title{
Layered Space-Time Multiuser Detection over Wireless Uplink Systems ${ }^{\ddagger}$
}

\author{
S. Sfar, R. D. Murch ${ }^{\dagger}$, and K. B. Letaief \\ Center for Wireless Information Technology \\ Electrical and Electronic Engineering Department \\ The Hong Kong University of Science \& Technology \\ Clear Water Bay, HONG KONG
}

\begin{abstract}
In this paper, we investigate the use of layered space-time, also known as Vertical-Bell Laboratories Layered Space-Time (V-BLAST) scheme, for multiuser detection in fading channels. The multiple transmit antennas in V-BLAST are treated as individual mobile station transmitters, while the base station consists of multiple receive antennas. In the proposed system, users are organized in groups and allocated a unique spreading code within the same group. Using these codes, we are able to separate the different groups, and layered space-time algorithm is then invoked to further remove the remaining interference between users. A decorrelator-type receiver based layered space-time detection is proposed for both complex and real constellations. For the latter case, we derive our receiver after evaluating and comparing the performance of two decorrelators based on the V-BLAST scheme. It is demonstrated that a significant performance improvement and increase in system capacity is obtained with very low spreading factors. Further results are also introduced by considering reduced complexity receivers based on Serial Layered Space-time Group Multiuser detection, and Parallel Layered Space-time Group Multiuser detection.
\end{abstract}

Keywords: Layered Space-Time Processing, Antenna Arrays, Multiuser Detection, Decorrelator-type Detectors.

\footnotetext{
$\dagger$ Corresponding Author: R. D. Murch. Email: eermurch@ee.ust.hk. Phone: (+852) 2335 7044. Fax: (+852) 2335 0194. Revised 25 March 2002.

$\ddagger$ this work is supported in part by the Hong Kong Research Grant council (Grant HKUST6060/99E) and the Hong Kong Telecom Institute of Information Technology.
} 


\section{INTRODUCTION}

Wireless communication systems have become very successful in the market place and it is expected that this success will continue when various interactive services and applications such as the Internet are introduced. The next generation of wireless networks will support multiple classes of traffic with different quality of service requirements such as data rate and bit error rate [1]. Hence, there has been a growing interest in using space domain processing for further improving the receiver performance and network capacity. Such processing has focused on the use of multiple antennas at the base station receiver to provide antenna gain and/or diversity gain $[2,3]$. By combining space-domain techniques with time domain techniques like multiuser detection [4, 5], the resulting space-time detectors [6]-[11] can further improve the system capacity over traditional time-domain-only detectors and enhance the quality of service provided to all the users. It has also been recently shown that the use of Multiple-Input Multiple-Output (MIMO) systems in a rich scattering wireless channel is capable of enormous theoretical capacity improvement and unprecedented spectral efficiencies over wireless channels [12, 13, 15]. Several architectures have been proposed to implement space-time systems for the MIMO flat fading wireless environment $[14,16]$. The vertical layered space-time, a single user MIMO architecture known as VBLAST, is one such approach, proposed by Foschini [14] to achieve high data rates and spectrum efficiency. Since its announcement, this system has gained considerable attention from both industry and academia. Recent work looked at further increasing the data rates, and thus, it was necessary to use signals whose bandwidth exceeds the coherence bandwidth of the channel, which brought in the issue of frequency selectivity. [17]-[20] proposed different approaches dealing and mitigating the effect of such wireless channels.

In contrast to previous work, we introduce in this paper the layered space-time scheme to multiuser detection. We consider, a system where users, each with a single antenna, transmit to a receiver with multiple antennas. In this system, users are organized in groups and allocated a unique spreading code within the same group. Using these codes, we are able to separate the different groups at the receiver. A generalization of layered space-time algorithm is then invoked to further remove the remaining interference between the users, and by doing so, a larger capacity in terms of the number of users can be achieved. The proposed system could be also seen as a multiuser MIMO system in which each user would carry more than one antenna for transmission. For example, we can consider that each group is one user with multiple transmit antennas. In this case, the network will present as many users as groups. With such a configuration, we are able to achieve very high data rates and spectral efficiencies by demultiplexing each user's input streams on the transmit antenna-array. High level modulations could be also used to further increase the network performance. Moreover, through the use of the spreading technique at the 
transmission, the proposed architecture inherits the ability to support CDMA applications, especially those proposed in the next generation CDMA systems. For instance, users could use variable spreading factors, constellations and powers for transmission, in order to increase their data rates and meet their QoS requirements.

To achieve good system performance, appropriate receivers structures must be designed. We propose in this paper various decorrelator-type receivers structure based on layered space-time detection. First, the transmitted informations are retrieved regardless of the groups from which they are sent. A significant performance improvement and increase in system capacity is obtained for very low spreading factors. We also explore enhancements to modulation based on the use of real constellation. Our approach shows that the limit of the channel capacity can be doubled for real constellations. We will provide in this paper a mathematical analysis of this case, and further simulation results will be given to demonstrate and verify the performance improvement. Then, we focus on reducing system computational complexity. To do so, we consider the application of the layered space-time algorithm within each group. Cases of serial and parallel multiuser detection are considered. It is shown that this complexity reduction in the system design will require an increase in the spreading factor to achieve better performance.

In [21], a decorrelating decision feedback multiuser detector for CDMA systems was proposed. When the number of users per group is one and the receiver presents only one antenna, the system described in [21] becomes very similar to the one presented here. This is because the decision feedback multiuser detector is almost the same as V-BLAST. The main difference between these two schemes is the interference cancellation ordering approach. The interested reader is referred to the work by G. Ginis and M. Cioffi [22], which compares the differences between V-BLAST and the system proposed in [21].

The organization of this paper is as follows. We provide in Section II, a system model and a full description of the layered space-time multiuser detection process. In Section III, we consider the special case of real constellations based modulations and derive two decorrelator-type detectors based on VBLAST. We then analyze their performance mathematically. The impact of these decorrelators on the proposed multiuser detector is also considered. In Section IV, we present various simulation results and discuss the application areas of the proposed systems. Further results are given in Section V where system complexity is reduced by proposing two other multiuser decorrelators based on serial and parallel group multiuser detection. We also investigate their performance using various parameters and analyze the tradeoff between bandwidth expansion and QOS requirements. Finally, Section VI concludes this paper. 


\section{LAYERED SPACE-TIME MULTIUSER DETECTORS}

The configuration of our system is shown in Figure 1 where signals from $K$ users, each with a single antenna, pass through a fading channel to a receiver with $P$ antennas. We associate each of the users to a group $g$ and in total there are $G$ groups. Each group has $M$ users so that the total number of users is $K=G \times M$. At a particular time instant, we let $\mathbf{b}=\left[b_{1}, \cdots, b_{K}\right]^{T}$ denote the $K \times 1$ symbol vector where each element represents the bit to be transmitted from a single mobile. These signals are spread using the $N \times K$ random spreading matrix $\mathbf{S}=\left[\mathbf{S}_{1} \cdots \mathbf{S}_{K}\right]$, where $\mathbf{S}_{k}$ is the $N \times 1$ code array for the $k$ th user. The chip values are $\pm 1 / \sqrt{N}$ and randomly generated with equal probability. We note that $S$ contains only $G$ different random spreading codes $\mathbf{S}_{1}, \cdots, \mathbf{S}_{G}$, each one is used by the $M$ users of its corresponding group. Hence, $\mathbf{S}=\left[\mathbf{S}_{1}, \cdots, \mathbf{S}_{1}, \mathbf{S}_{2}, \cdots, \mathbf{S}_{2}, \cdots, \mathbf{S}_{G}, \cdots, \mathbf{S}_{G}\right]$. These signals pass through the fading channel that we assume to be frequency non-selective, to the base station, and we write the channel matrix as $\mathbf{H}=\left[\mathbf{h}_{1} \cdots \mathbf{h}_{P}\right]$ where $\mathbf{h}_{p}$ is the $1 \times K$ channel coefficient vector between the $p$ th receive antenna and all $K$ users.

The received baseband signal for a given symbol period at the $p$ th antenna, following a chip-matched filter, can then be written as a complex $N$-vector

$$
\mathbf{r}_{\mathbf{p}}=\mathbf{S C}_{p} \mathbf{b}+\mathbf{n}_{\mathbf{p}}
$$

where $\mathbf{C}_{p}$ denotes the complex diagonal channel matrix for the $p$ th antenna, $\operatorname{diag}\left(\mathbf{h}_{p}\right)$. The noise vector $\mathbf{n}_{\mathbf{p}}$ is a complex-valued zero-mean Gaussian random $N$-vector with a covariance matrix $\sigma^{2} I_{N}$, in which $I_{N}$ denotes the $N \times N$ identity matrix.

For simplicity, transmitted data is radiated using the same constellation, spreading factor $N$, and power for transmission. In addition, antennas for transmissions, or users, are assumed to be far enough form each other, such that channel links are independent. Perfect channel estimation and symbol synchronization, are also assumed. We note that [23] details the impact of asynchronous transmission, and similar approaches could be used in such case for our system.

\section{A. Receiver Structure}

We consider a decorrelator-type receiver [10] based layered space-time detection scheme as a spacetime multiuser detection in the uplink of transmission systems as shown in Figure 1. The detection process consists of performing a combined space code matched filtering operation to separate between the groups. Layered space-time decorrelation is then invoked in order to eliminate the remaining interuser interference. Users are ranked according to their SNR's following the space-code matched filter. This implies that two successive detected users do not have to belong to the same group. The considered receiver structure consists, thus, of a bank of $P$ correlators matched to the $K$ path spreading codes. 


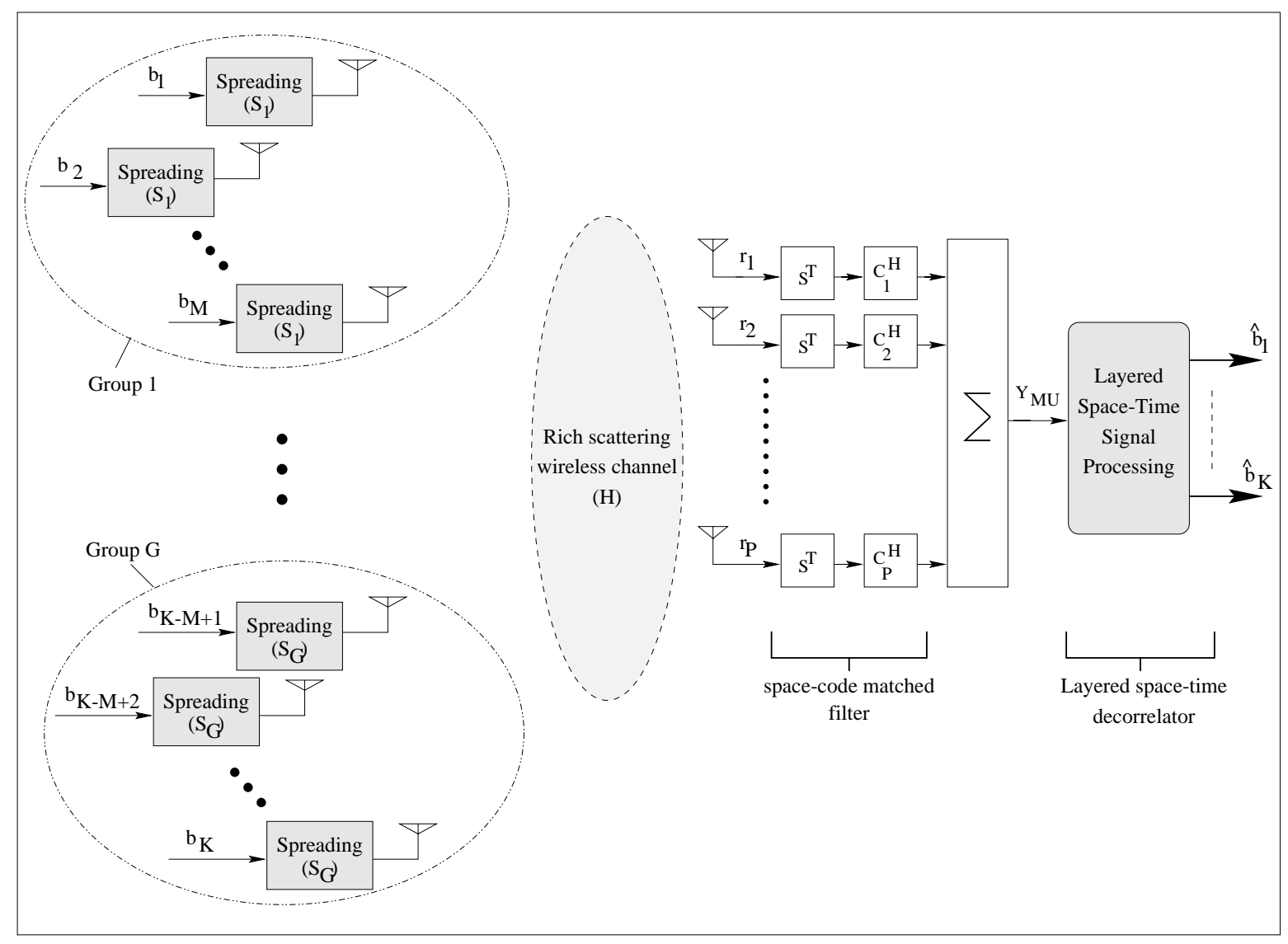

Figure 1: Layered Space-time Multiuser system block diagram

The correlator output for the $k$ th user at the $p$ th antenna is thus weighted by the conjugate of the array/channel coefficient, assuming perfect channel estimation.

Let $\mathbf{Y}_{\mathrm{MU}}$ be the sufficient statistic $K$ vector obtained after the space-code matched filter with respect to the codes and the channel coefficients [10]. $\mathbf{Y}_{\mathbf{M U}}$ can be written as

$$
\mathbf{Y}_{M U}=\sum_{p=1}^{P} \mathbf{C}_{p}^{H} \mathbf{S}^{T} \mathbf{r}_{p}=\tilde{\mathbf{R}}_{M U} \mathbf{b}+\tilde{\mathbf{n}}
$$

where $\tilde{\mathbf{R}}_{M U}=\sum_{p=1}^{P} \mathbf{X}_{p}^{H} \mathbf{X}_{p}$ is the $K \times K$ space-code cross-correlation matrix in which $\mathbf{X}_{p}=\mathbf{S C}_{p}$. $\tilde{\mathbf{n}}=\sum_{p=1}^{P} \mathbf{X}_{p}^{H} \mathbf{n}_{p}$ is a real $K$ Gaussian noise vector with covariance $\sigma^{2} \tilde{\mathbf{R}}_{M U}$.

\section{B. Detection Algorithm}

The detection algorithm is based on an iterative process and we use $i$ to denote the $i$ th iteration. The received vector is indexed by $i$ to give $\mathbf{r}_{p}(i)$ and similarly the space-code cross-correlation channel matrix $\tilde{\mathbf{R}}_{M U}(i)$ and the sufficient statistic vector $\mathbf{Y}_{M U}(i)$. The iterative process consists of three steps; namely, the computation of the nulling vector, signal estimation, and interference cancellation.

The first step consists of computing the pseudo-inverse [25] of the space-code cross-correlation matrix, $\left[\tilde{\mathbf{R}}_{M U}(i)\right]^{+}$. Users are then ranked according to their post detection SNR's. The user with the highest 
SNR, defined by

$$
k_{i}=\arg \max _{j \notin\left\{k_{1} \ldots k_{i-1}\right\}} \frac{\left\|b_{j}\right\|^{2}}{\sigma^{2}\left[\tilde{\mathbf{R}}_{M U}(i)^{+}\right]_{(j, j)}}
$$

is then selected. The notation " $\mathbf{R}_{(i, j)}$ " denotes the element of the matrix $\mathbf{R}$ at the $i$ th row and $j$ th column, and "|l.|", the conventionnal 2-norm [26]. We note that, since all users have the same transmission power,

$$
k_{i}=\arg \min _{j \notin\left\{k_{1} \ldots k_{i-1}\right\}}\left[\tilde{\mathbf{R}}_{M U}(i)\right]_{(j, j)}^{+} .
$$

The nulling vector corresponding to this user is the $k_{i}$ th column of $\left[\tilde{\mathbf{R}}_{M U}(i)\right]^{+}$, written as $\mathbf{w}_{k_{i}}=$ $\left[\tilde{\mathbf{R}}_{M U}(i)\right]_{k_{i}}^{+}$. The slicer output vector, $z_{k_{i}}$, and the estimated symbol, $\hat{b}_{k_{i}}$, are computed such that

$$
\begin{array}{r}
z_{k_{i}}=\mathbf{w}_{k_{i}}^{T} \mathbf{Y}_{M U}(i) \\
\hat{b}_{k_{i}}=\Psi\left(z_{k_{i}}\right) .
\end{array}
$$

Finally, interference cancellation is performed and the detected symbol is taken out to give

$$
\mathbf{r}_{p}(i+1)=\mathbf{r}_{p}(i)-\left(\mathbf{X}_{p}(i)\right)_{k_{i}} \hat{b}_{k_{i}}
$$

$\mathbf{X}_{p}(i+1)$ and $\tilde{\mathbf{R}}_{M U}(i+1)$ are obtained, respectively, by striking out the $k_{i}$ th column of $\mathbf{X}_{p}(i)$ and the $k_{i}$ th row and column of $\tilde{\mathbf{R}}_{M U}(i)$. The sufficient statistic vector for the $(i+1)$ iteration is then defined by

$$
\mathbf{Y}_{M U}(i+1)=\sum_{p=1}^{P} \mathbf{X}_{p}(i+1)^{H} \mathbf{r}_{p}(i+1)
$$

$\mathbf{Y}_{M U}(i+1)$ could be also expressed as $\left[\mathbf{Y}_{M U}(i)-\left(\tilde{\mathbf{R}}_{M U}(i)\right)_{k_{i}} \hat{b}_{k_{i}}\right]^{\overline{k_{i}}}$, where "(.) $\bar{j}$ " denotes the operation of striking out the $j$ th row of the matrix "(.)". This iterative process is continued until all $K$ signals are detected.

\section{LAYERED SPACE-TIME MULTIUSER DETECTION FOR REAL CONSTELLATIONS}

For the particular case of real constellations, some special properties are of interest and these lead to a doubling of the expected system capacity and spectrum efficiency. We consider here the case of synchronous BPSK data signals and denote by "r" the "real" operator used to retrieve the real part of the data constellation, and by "T" the transformation used to detect the transmitted signals. In this paper, the operator " $\mathbf{T}$ " is non-linear and consists of the layered space-time signal processing described in Section II.

We are interested in the effect of the order of the real operator $\mathbf{r}$ and the transformation $\mathbf{T}$ on system performance. Specifically, we wish to know if we should use the operator $\mathbf{r}$ before or after $\mathbf{T}$. In other words, should we consider the real part of the sufficient statistic vector before applying the 
layered space-time or only at the level of the decision function $\Psi($.$) , defined in (6)? We consider both$ cases where $\mathbf{r}$ precedes $\mathbf{T}$ and the opposite structure, denoted respectively by the subscript "rT" and "Tr". For simplicity, we consider the same system configuration as in Section II, but with only one group of users. In this case, spreading is ignored and receivers are equivalent to two decorrelators based on layered space-time as shown in Figure 2, and denoted Tr-BLAST and rT-BLAST, respectively. In Appendix A, we prove this equivalence for the case of the rT-type receiver. A similar proof could be done in the case of the Tr-type receiver. For more details, the reader is referred to Appendix A.

The above receivers, and in contrast to previous work [14], present a space matched filter followed by a layered space-time unit. Using the notations introduced in Section II, the received vector $r$ satisfies the following equality

$$
\mathbf{r}=\mathbf{H b}+\mathbf{n}
$$

in which $\mathbf{n}$ is a Gaussian noise vector with zero mean and $\sigma^{2} \mathbf{I}_{\mathbf{M}}$ as a covariance. The sufficient statistic vector, $\mathbf{Y}_{\mathbf{T r}}$, for $\mathbf{T r}$-BLAST satisfies

$$
\begin{aligned}
\mathbf{Y}_{\operatorname{Tr}} & =\mathbf{H}^{\mathbf{H}} \mathbf{r} \\
& =\mathbf{R}_{\operatorname{Tr}} \mathbf{b}+\tilde{\mathbf{n}}_{\operatorname{Tr}}
\end{aligned}
$$

where $\mathbf{R}_{\operatorname{Tr}}$ is the channel cross-correlation matrix and $\tilde{\mathbf{n}}_{\operatorname{Tr}}$ is a complex-valued zero-mean Gaussian random noise-vector with covariance matrix $\sigma^{2} \mathbf{R}_{\operatorname{Tr}}$. The Tr-BLAST detection algorithm consists of the same steps and operations as in Section III.B.2, using as a cross-correlation $\mathbf{R}_{\operatorname{Tr}}(j)=\mathbf{H}(j)^{H} \mathbf{H}(j)$ for the $j$ th iteration. The sufficient statistic vector is then defined for the next iteration by

$$
\mathbf{Y}_{\operatorname{Tr}}(j+1)=\mathbf{H}(j+1)^{H} \mathbf{H}(\mathbf{b}-\hat{\mathbf{b}}(j))+\mathbf{H}(j+1)^{H} \mathbf{n}
$$

where $\hat{\mathbf{b}}(j)$ denotes the estimated vector of $\mathbf{b}$ at the $j$ th iteration and contains all the previously estimated bits, $\left\{\hat{b}_{k_{1}}, \cdots, \hat{b}_{k_{j-1}}\right\}$.

The sufficient statistic vector, $\mathbf{Y}_{\mathbf{r T}}$, for the $\mathbf{r T}$-BLAST decorrelator is given by

$$
\mathbf{Y}_{\mathbf{r T}}=\operatorname{Re}\left(\mathbf{R}_{\mathbf{T r}} \mathbf{b}+\tilde{\mathbf{n}}_{\mathbf{T r}}\right)=\mathbf{R}_{\mathbf{r T}} \mathbf{b}+\tilde{\mathbf{n}}_{\mathbf{r} \mathbf{T}}
$$

where $\tilde{\mathbf{n}}_{\mathbf{r T}}$ is a real-valued zero-mean Gaussian random noise-vector with covariance matrix $\sigma^{2} \mathbf{R}_{\mathbf{r T}}$. Thus, at each iteration $j, \mathbf{R}_{\mathbf{r T}}(\mathbf{j})^{(M \times M)}$ is equal to $\operatorname{Re}\left(\mathbf{H}(j)^{H} \mathbf{H}(j)\right)$.

To compare the two approaches, we first consider the range $0<M \leq P$. Assume $\left\{k_{1}, \cdots, k_{M}\right\}$ and $\left\{k_{1}^{\prime}, \cdots, k_{M}^{\prime}\right\}$ be the optimal detection order for $\operatorname{Tr}$ and $\mathbf{r T}$-BLAST respectively, and denote their overall BER by $B E R_{\operatorname{Tr}}$ and $B E R_{\mathbf{r T}}$. It follows that $B E R_{\mathbf{r T}}\left(\left\{k_{1}^{\prime}, \cdots, k_{M}^{\prime}\right\}\right)$ and $B E R_{\operatorname{Tr}}\left(\left\{k_{1}, \cdots, k_{M}\right\}\right)$ are the minimum possible probability of error achievable by such receivers [15]. To show that the rT-BLAST 


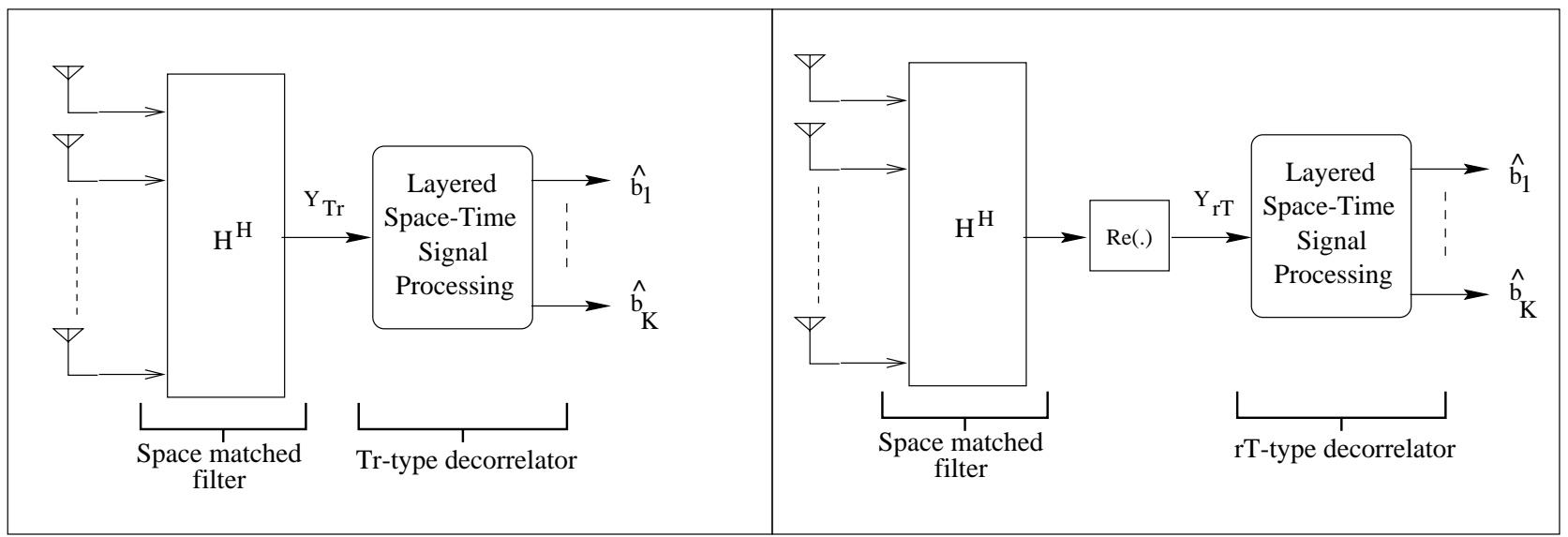

a)

b)

Figure 2: Block diagram of: a) Tr-BLAST decorrelator b) rT-BLAST decorrelator

decorrelator outperforms the $\operatorname{Tr}$ decorrelator in terms of the overall achieved BER when $0<M \leq P$, we will prove the following

$$
B E R_{\mathbf{r} \mathbf{T}}\left(\left\{k_{1}^{\prime}, \cdots, k_{M}^{\prime}\right\}\right) \leq B E R_{\mathbf{r T}}\left(\left\{k_{1}, \cdots, k_{M}\right\}\right) \leq B E R_{\operatorname{Tr}}\left(\left\{k_{1}, \cdots, k_{M}\right\}\right),
$$

in which $B E R_{\mathbf{r T}}\left(\left\{k_{1}, \cdots, k_{M}\right\}\right)$ is the overall BER obtained by $\mathbf{r T}$-BLAST using the optimal detection order of Tr-BLAST. The left part of this inequality is therefore immediate since $\left\{k_{1}, \cdots, k_{M}\right\}$ is not the optimal order in the $\mathbf{r} \mathbf{T}$ receiver sense.

Next, in order to prove the right inequality, we evaluate the bit error rate for each receiver at each iteration. Let us consider first the Tr-BLAST decorrelator. The slicer input for the $j$ th iteration satisfies

$$
\begin{aligned}
z_{k_{j}} & =\mathbf{w}_{k_{j}}^{T} \mathbf{Y}_{\operatorname{Tr}}(j) \\
& =\mathbf{w}_{k_{j}}^{T} \mathbf{H}(j)^{H} \mathbf{H}(\mathbf{b}-\hat{\mathbf{b}}(j))+\mathbf{w}_{k_{j}}^{T} \mathbf{H}(j)^{H} \mathbf{n} \\
& =\mathbf{w}_{k_{j}}^{T} \mathbf{H}(j)^{H} \mathbf{H}(\mathbf{b}-\hat{\mathbf{b}}(j))+\nu_{k_{j}} .
\end{aligned}
$$

The noise, $\nu_{k_{j}}$, in this component is Gaussian distributed with $N\left(O, \sigma^{2}\left[\mathbf{R}_{\operatorname{Tr}}(j)\right]_{\left(k_{j}, k_{j}\right)}^{+}\right)$. Thus, the BER for the $k_{j}$ user is given by [10]

$$
B E R_{\operatorname{Tr}, k_{j}}=Q\left[\sqrt{\frac{\left|\left(\left[\mathbf{R}_{\operatorname{Tr}}(j)\right]^{+} \mathbf{H}(j)^{H} \mathbf{H}(\mathbf{b}-\hat{\mathbf{b}}(j))\right)_{k_{j}}\right|^{2}}{\sigma^{2}\left[\mathbf{R}_{\operatorname{Tr}}(j)\right]_{\left(k_{j}, k_{j}\right)}^{+}}}\right]
$$

where $Q[x]=\frac{1}{\sqrt{2 \pi}} \int_{x}^{\infty} e^{-\frac{t^{2}}{2}} d t$.

Similarly, the BER of the $k_{j}$ th detected user with the rT-BLAST decorrelator, is estimated by the 
following equation

$$
B E R_{\mathbf{r} \mathbf{T}, k_{j}}=Q\left[\sqrt{\frac{\left|\left(\left[\mathbf{R}_{\mathbf{r} \mathbf{T}}(j)\right]^{+} \mathbf{H}(j)^{H} \mathbf{H}(\mathbf{b}-\hat{\mathbf{b}}(j))\right)_{k_{j}}\right|^{2}}{\sigma^{2}\left[\mathbf{R}_{\mathbf{r} \mathbf{T}}(j)\right]_{\left(k_{j}, k_{j}\right)}^{+}}}\right]
$$

Recall that the only difference between these two decorrelators is that the order of the real operator and the non-linear transformation are interchanged. Hence, the BER of the rT-BLAST decorrelator for the $k_{j}$ th bit satisfies the following equality

$$
\begin{aligned}
B E R_{\mathbf{r} \mathbf{T}, k_{j}} & =Q\left[\sqrt{\left.\frac{\left|\left(\left[\mathbf{R}_{\mathbf{r} \mathbf{T}}(j)\right]^{+} \mathbf{H}(j)^{H} \mathbf{H}(\mathbf{b}-\hat{\mathbf{b}}(j))\right)_{k_{j}}\right|^{2}}{\sigma^{2}\left[\mathbf{R}_{\mathbf{r} \mathbf{T}}(j)\right]_{\left(k_{j}, k_{j}\right)}^{+}}\right]}\right. \\
& =Q\left[\sqrt{\frac{\left|\left(\left[\operatorname{Re}\left(\mathbf{R}_{\operatorname{Tr}}\right)(j)\right]^{+} \mathbf{H}(j)^{H} \mathbf{H}(\mathbf{b}-\hat{\mathbf{b}}(j))\right)_{k_{j}}\right|^{2}}{\sigma^{2}\left[\operatorname{Re}\left(\mathbf{R}_{\mathbf{T r}}\right)(j)\right]_{\left(k_{j}, k_{j}\right)}^{+}}}\right]
\end{aligned}
$$

To prove the right inequality in (13), we show in Appendix $\mathrm{C}$ that at any iteration $j$, the $\mathbf{r T}$ receiver would provide better performance for each user than Tr-BLAST, when using the same order of detection.

$$
\forall j \in\{1, \cdots, M\} ; \quad B E R_{\mathbf{r T}, k_{j}} \leq B E R_{\operatorname{Tr}, k_{j}}
$$

Intuitively, it is clear that taking the real part of a signal corrupted by a complex noise has to reduce the level of interference and improve the resultig BER. It is immediate that in such a case the rT receiver would outperform the $\operatorname{Tr}$ detector in terms of the overall BER. However, under imperfect interference cancellation the proof complexity is very high, and for simplicity we assume perfect interference cancellation. For more details, the reader is referred to Appendix C.

Next, for the second range $P<M \leq(2 P)$, note that Tr-BLAST will not function because its crosscorrelation matrix is singular. However, the $\mathbf{r} \mathbf{T}$ receiver could be deployed because $\mathbf{R}_{\mathbf{r}} \mathbf{T}$ is non-singular in this case. This is due to the rich scattering environment and the antennas separation, so the complex columns of the matrix $\mathbf{H}^{(P \times M)}(j)$ are real-linearly independent. Appendix B shows in general that for any real-linearly independent complex matrix $\mathbf{F}^{n \times m}$, the matrix $\mathbf{F}^{H} \mathbf{F}$ is invertible only if $n \geq m$, and $\operatorname{Re}\left(\mathbf{F}^{H} \mathbf{F}\right)$ is invertible only if $(2 n) \geq m$. Therefore, for the special case of real constellations, we propose to apply the Layered Space-Time scheme described in Section II to the real part of the sufficient statistic vector in order to obtain a better BER performance. This will also allow us to double the network capacity in terms of the number of users and hence system spectrum efficiency. A direct result, will be to double the number of users in the case of BPSK modulation and thus achieve the same system spectral efficiency as with 4-QAM. The result, in fact, confirms the work in [24]. 


\section{SIMULATION RESULTS}

We investigate here the performance of the proposed multiuser detector. We first consider the effect of the grouping and spreading factors for the LAyered Space-Time Multiuser Detector (LAST-MUD) using 4-QAM modulation. Then, we increase the system capacity by increasing the number of groups for a fixed configuration. Next, and in the rest of the section we consider BPSK modulation to demonstrate the performance of real constellations. We compare the rT and Tr-BLAST decorrelator performances, in order to confirm the conclusions of Section III. C. Then, we provide simulation results for LAST-MUD based on the rT-type decorrelator with BPSK modulation.

Throughout this section, we assume that the number of receive antennas per base station is fixed to 6 , and the number of users and groups are varied.

\section{A. LAST-MUD Performance with Complex Constellations}

We analyze here the symbol error rate (SER) of LAST-MUD with 4-QAM modulation, using a total of 12 users. Figure 3 presents the effect of grouping on the system performance for a fixed spreading factor $N$ equal to 15. It is clear that when all the users have the same code, or share the same group, our system performance is equivalent to V-BLAST (See Appendix A). Hence, we should have $P \geq K$ or $M$ in this case; otherwise the receiver does not provide enough degree of freedom to separate between the coming signals. If $K \geq P$, which is the case in Figure 3 when $M=12$, the performance is very bad and the BER is about 0.5. Hence, to accommodate 12 users, we split them equally to $2,3,4$ and 6 groups, so that we can use spreading to separate between the groups and layered space-time to increase the capacity. From the simulation results, we conclude that for fixed numbers of users, the larger the number of groups $G$, the better the performance, since more codes are being deployed. For example, the SER is limited to about $3 \times 10^{-1}$ when $G=1$. However, at $10 \mathrm{~dB}$ SNR with 3 groups, we achieve an SER of $2 \times 10^{-5}$. Hence, with even a small spreading factor, such as 15 , we can achieve a gain of the order of $10^{-4}$ in the SER performance.

The second step consists of analyzing the effect of the spreading factor $N$. For this purpose, we consider a fixed configuration of 3 groups of 4 users each, and we vary $N$ to have the following values 8, 10, 12, 15 and 20. Figure 4.a) shows the SER variation. With a spreading factor of 8 , which represents $67 \%$ of the users, we achieve a probability of error of the order $10^{-3}$ at $10 \mathrm{~dB}$. A probability of almost $10^{-5}$ is achieved when $N$ is equal to 12 at $10 \mathrm{~dB}$ SNR. On the other hand, we notice that the performance achieved with $N$ equal to 15 is the same as that of one group of 4 users. Hence, with $N=15$, the system of 3 groups of 4 users achieves a single group of 4 users performance. We notice also in Figure 4.a), that when $N=20$ we achieve the same SER limit. Thus, a spreading factor of 15 is enough to achieve the single group performance. As a result, a higher bandwidth expansion is not needed. We also analyze 


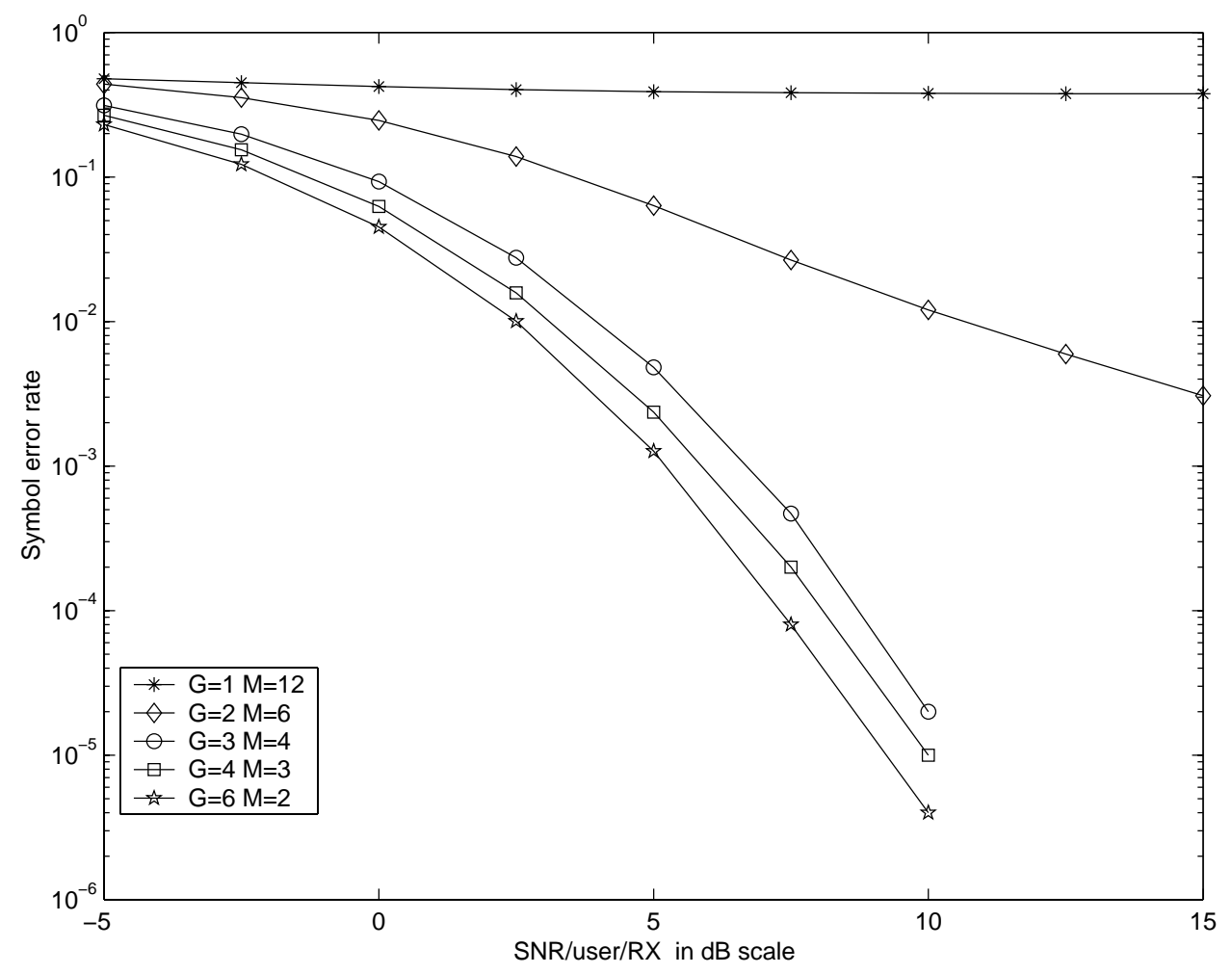

Figure 3: Grouping effect on LAST-MUD for 4-QAM with 12 users, 6 RX and $N=15$

system behavior with larger processing gains and number of users. We consider a configuration of 25 groups of 4 users each, with $N=32,64$ and 128. Figure 4.b) shows that we have the same conclusions with large and small spreading factors. We notice that with $N=32$, there is almost $1 \mathrm{~dB}$ loss in SNR compared to the single group performance. However, the latter is fully achieved with $N=64$ and 128 . Hence, a spreading factor of the order of $32 \%$ of the number of users provides SER ranges very close to the single group case. A direct consequence is to extend these results to the special case where each user has its own code, or that different groups consist of a single user. With such a scheme, the performance of multiuser systems could achieve the single user system performance with low spreading factors.

In order to increase the number of users in the network, we fix the spreading factor to 15 and the number of users per group to 4 . Then, we increase the number of groups to $3,6,9$ and 12 . We present the SER results in Figure 5. We notice that with LAST-MUD, we can achieve an SER of the order of $10^{-5}$ for 36 users at an SNR of $10 \mathrm{~dB}$, or $10^{-4}$ for 48 users. We would like to note also that in Figure 4.b), we show that 25 groups could be accommodated with $N=32$ achieving almost the optimum performance. Hence, LAST-MUD provides very high network capacities with very low spreading factors, and this can have a significant practical impact. 

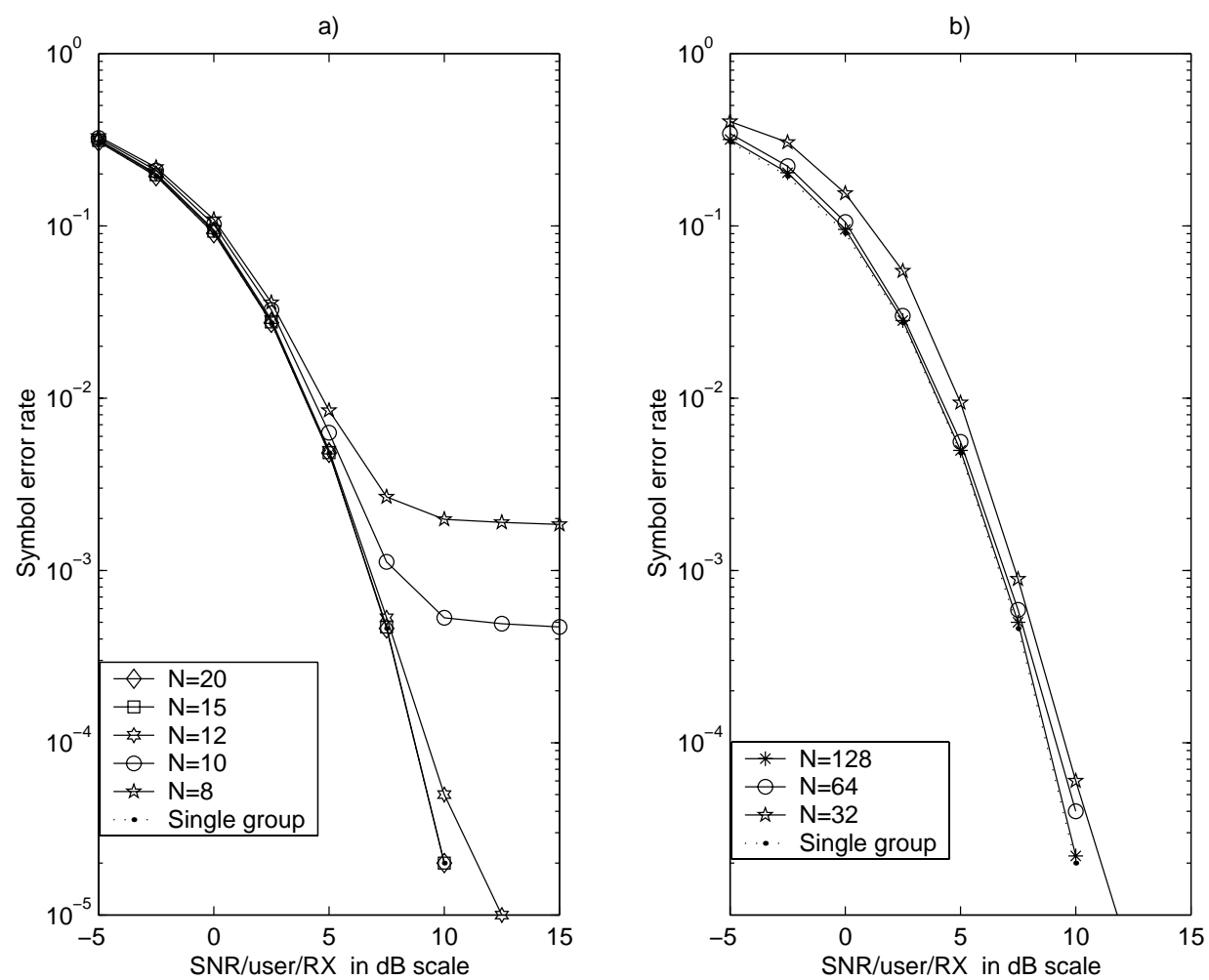

Figure 4: Spreading effect on LAST-MUD for 4-QAM with 6 RX and a) 3 groups of 4 users, b) 25 groups of 4 users

\section{B. rT and $\mathbf{T r}-B L A S T$ decorrelator Performance Comparison with Real Constellations}

We focus here on the real constellation case. Although we proved in Section IV.C that $\mathbf{r T}$ detector outperforms the $\operatorname{Tr}$ receiver, we would like to observe these results by simulations. Hence, we compare the rT and Tr-BLAST decorrelators performance using simulation results for point to point communication systems, but with multiple transmit and receive antennas and BPSK modulation. Figure 6 shows the BER of both detectors using 6 receive antennas and 4, 8, 10, 12 and 15 transmit antennas, respectively. The horizontal axis is the average spatial received signal-to-noise ratio for each transmit antenna. As explained in Section IV.C, it is expected that the $\mathbf{T r}$ receiver performance drops dramatically when the number of transmit antennas $M$ is larger than the number of receiver antennas $P$. This is explained by the fact that the receiver does not provide enough degrees of freedom to separate the coming transmitted signals, and hence, the cross-correlation matrix $\mathbf{R}_{\operatorname{Tr}}$ is singular (See Appendix A). This phenomenon is observed in Figure 6, where we notice that the BER for (8TX, 6RX) is bounded by $10^{-2}$. In contrast, rT receiver achieves good ranges of BER such as $10^{-6}$ at $7.5 \mathrm{~dB}$ SNR. In addition, the BER gap between these two receivers is huge when 10 transmitter are deployed. But, as Figure 6 shows, when $M>2 P$, BER performance drops since $\mathbf{R}_{\mathbf{r}} \mathbf{T}$ is singular (See Appendix B). This shows how system capacity is doubled via the use of the rT receiver while keeping low ranges of BER. Intuitively, ignoring the imaginary data contribution while processing the layered space-time detection minimizes the noise corruption and 


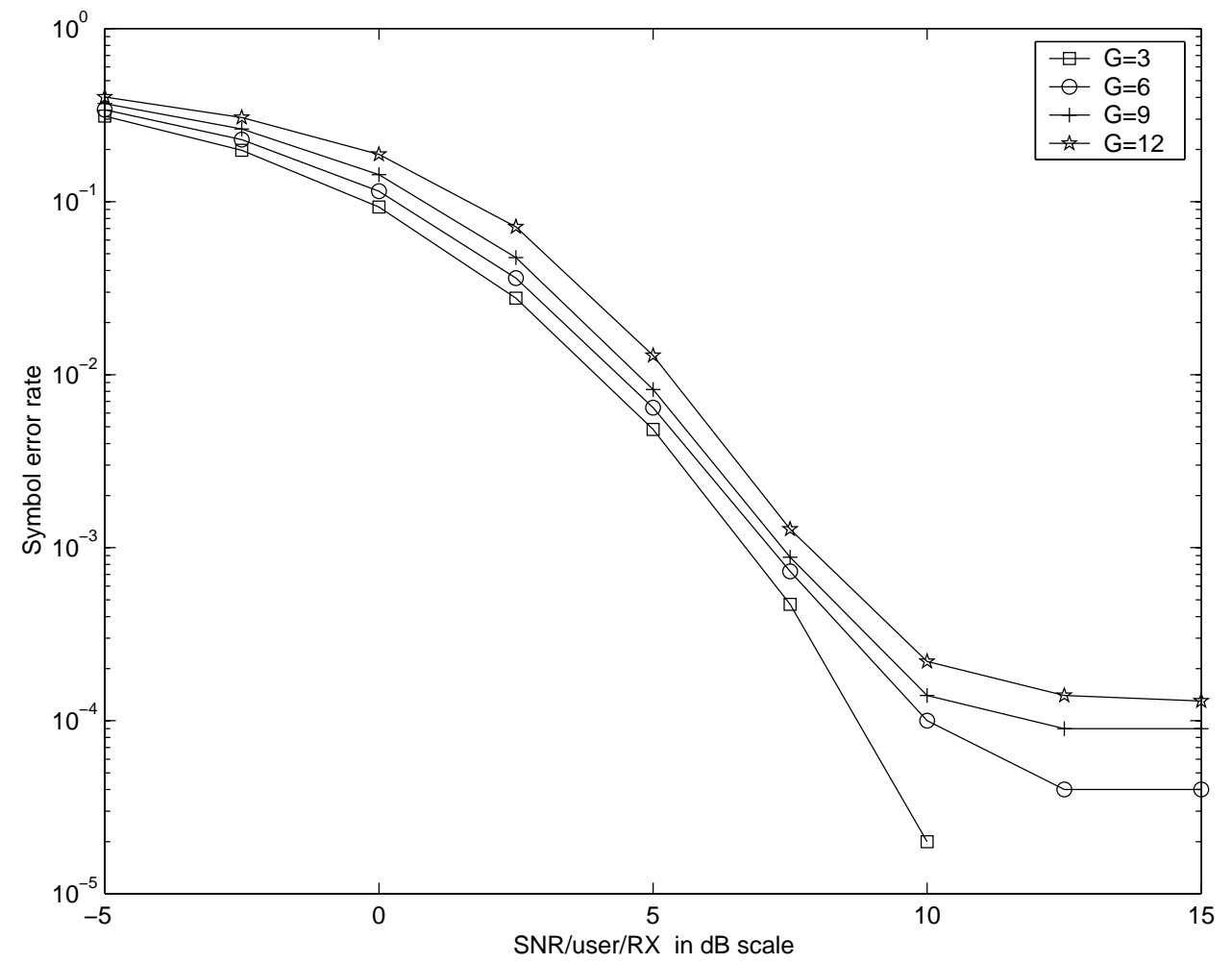

Figure 5: Increasing the network capacity using LAST-MUD with 4-QAM, $6 R X, M=4$ and $N=15$

optimizes the ordering, when real constellations are used. Finally, we would like to note that rT-BLAST curves represent the performance limit achieved by LAST-MUD receiver. In fact, as shown in Appendix A, the performance of a single group of $M$ users with LAST-MUD system is the same as the performance of rT with $M$ transmit antennas. Therefore, when we increase the spreading factor in LAST-MUD, as we did in Section IV.A (See Figure 4), we achieve the performance limit.

\section{LAST-MUD Performance with Real Constellations}

We consider here LAST-MUD performance for BPSK modulation based on the rT-BLAST decorrelator. As in the case of the previous analysis with 4-QAM, we investigate the effect of grouping. We fix the number of users to 12, the spreading factor to 15 and divide the users into 2, 3, 4 and 6 groups. We found that grouping has the same effect on BER variations for real constellations as for complex constellations. For example, we found that a gain of the order of $10^{-4}$ at an SNR of $2.5 \mathrm{~dB}$ is achieved with 2 groups. A same order of gain is also obtained with 3,4 and 6 groups. We also investigate in Figure 7 the performance of this system while increasing the number of groups with $N=15$ for a fixed number of users per group $(M=10)$. We notice that a two group performance is the same as the single group case. The latter is also equivalent to the performance of the rT-BLAST decorrelator with 10 TX and 6 RX (See Figure 6), as explained in Section IV.B. Hence, we conclude that with a spreading factor of 15 , we achieve the single group performance for 20 users divided in 2 groups of 10, as shown 


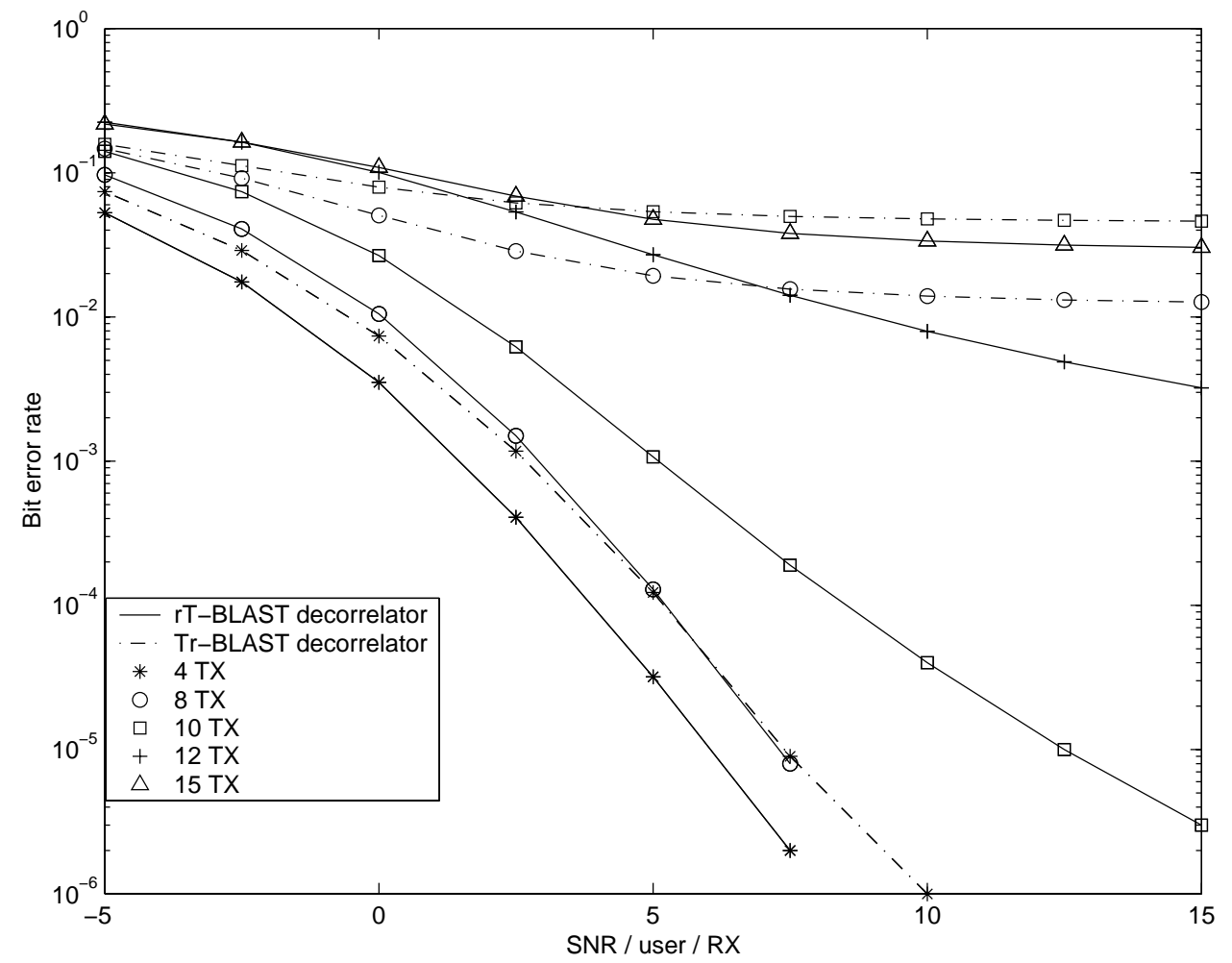

Figure 6: Performance comparison between the $\mathbf{r T}$ and the $\operatorname{Tr}$-BLAST decorrelator with 6 RX and BPSK

in Appendix B. We also show in Figure 7 that we can further increase the network capacity to 50 users. For example, considering a spreading factor of 15 and an SNR of $15 \mathrm{~dB}$, a BER of the order of $10^{-5}$ is achieved. For complex constellations, the LAST-MUD permits us to achieve very high network capacities with very low spreading factors. High data rates and spectral efficiencies could be also obtained, and delay sensitive applications are very well served with this receiver. The performance achieved with the proposed scheme presents a significant improvement compared to traditional uncoded systems.

\section{REDUCED COMPLEXITY LAYERED SPACE-TIME GROUP MULTIUSER DETECTION}

The computational complexity of our proposed LAST-MUD receiver, as for any decorrelator-type detector, is high due to the inversion of the space-code cross-correlation matrix. In order to reduce system complexity, we propose in this section to apply a layered space-time technique to each group instead of all users as for LAST-MUD. The receiver structure will then present $G$ blocks of layered space-time signal processing units corresponding to the $G$ groups of users. To present this technique, we introduce some additional notations; namely, $\mathbf{C}_{g_{p}}=\operatorname{diag}\left(\left[h_{p,(g-1) M+1} \cdots h_{p, g M}\right]\right)$, the complex $M \times M$ diagonal channel coefficient matrix between the $g$ th group and the $p$ th receive antenna, and $\mathbf{S}^{(g)}=\left[\mathbf{S}_{(g-1) M+1} \cdots \mathbf{S}_{g M}\right]$ the $N \times M$ spreading matrix for the $g$ th group. Similarly, we define $\mathbf{X}_{g_{p}}=\mathbf{S}^{(g)} \mathbf{C}_{g_{p}}$ the combined channelcode matrix for antenna $p$. The remaining notations and assumptions defined in Section II remain valid, and the received vectors by each antenna still satisfy (1). Two possible receiver structures are given as 


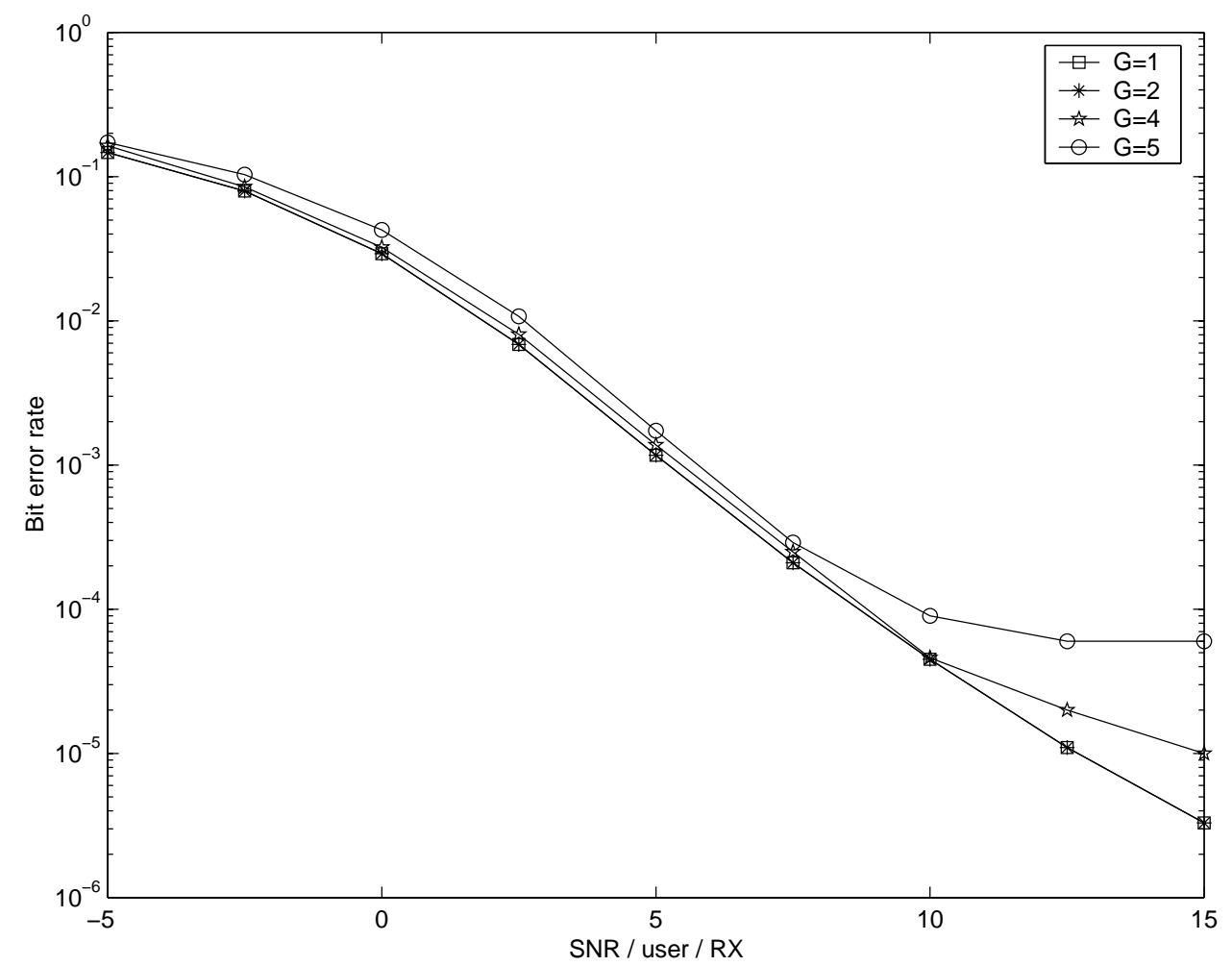

Figure 7: Increasing the network capacity using LAST-MUD with BPSK, 6 RX, M=10 and $N=15$

described next.

\section{A. Serial-Layered Space-time Group Multiuser Detector}

Inspired by the serial interference cancellation [5], we propose a Layered Space-time Group Multiuser Decorrelator with Serial Group Interference Cancellation that we will denote by Serial LAyered SpaceTime Group MUltiuser Detector or Serial LASTG-MUD. A block diagram of this receiver structure is given in Figure 8. The latter is composed of $G$ branches connected serially. Each branch is dedicated to one group, and is composed of a group space-code unit (See Fig. 8.b)) followed by a layered space-time signal processing unit. The first unit consists of despreading the group code, then matching the receiver to the channel seen by the corresponding group. The layered space-time unit will then extract the signal data transmitted by the considered group, and the detected set of data will be removed from the sufficient statistic vector of the remaining groups. This process will continue until all data are extracted.

As in the case of LAST-MUD, the detection process consists of first setting the group counter, $g$, to 0 and the estimated signal vector, $\hat{\mathbf{b}}$, to $\mathbf{0}_{1 \times K}$ since no signal has been already detected yet. Then, the different groups of users are treated linearly in different branches each, and each group's signals set is detected in a similar way as described in Section II.B. Hence, a group initialization and a group iterative process are defined. In the first part, the group counter $g$ is updated, $g=g+1$. The group iteration 


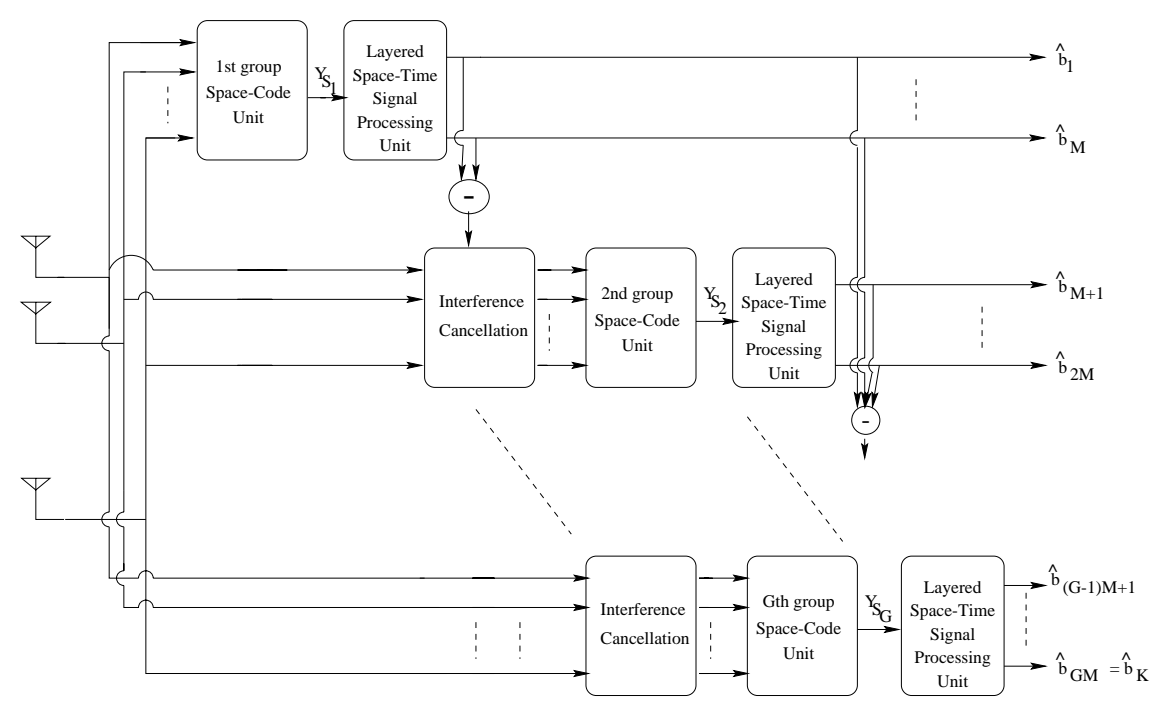

a)

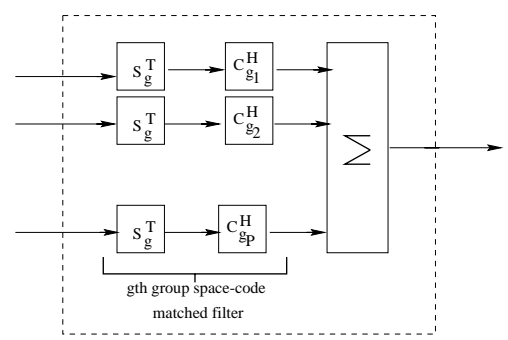

b)

Figure 8: a) Serial-Layered Space-Time Group Multiuser Detector b) The $g^{\text {th }}$ group Space-Code Unit

index, $i$, is also initialized to 1 . The group space-code cross-correlation matrix is computed as

$$
\tilde{\mathbf{R}}_{\mathbf{S}}^{(g)}=\sum_{p=1}^{P} \mathbf{X}_{g_{p}}^{H} \mathbf{X}_{g_{p}} .
$$

The group vector input to the $g$ th group space-code unit and corresponding to the $p$ th receive antenna is given by $\mathbf{r}_{g_{p}}=\mathbf{X}_{p}(\mathbf{b}-\hat{\mathbf{b}})+\mathbf{n}_{p}$. The estimated sets of data of the previous detected groups are thus removed serially. The group sufficient statistic vector is defined by

$$
\mathbf{Y}_{\mathbf{S}_{\mathbf{g}}}=\sum_{p=1}^{P} \mathbf{X}_{g_{p}}^{H} \mathbf{r}_{g_{p}}
$$

The next step consists of a group iterative process which consists of three steps as described before in Section II.B. First, the nulling vector, $\mathbf{w}_{k_{i}}$, is obtained from the pseudo-inverse of $\tilde{\mathbf{R}}_{\mathbf{S}}^{(g)}(i)$ of each iteration. Second, and after the slicing operation, $z_{k_{i}}=\mathbf{w}_{k_{i}}^{T} \mathbf{Y}_{\mathbf{S}_{\mathbf{g}}}(i)$, the $k_{i}$ th signal of the $g$ th group is estimated

$$
\hat{b}\left((g-1) \times M+k_{i}\right)=\Psi\left(z_{k_{i}}\right) .
$$

Finally, the estimated signal interference is removed, and the sufficient statistic vector of the next 


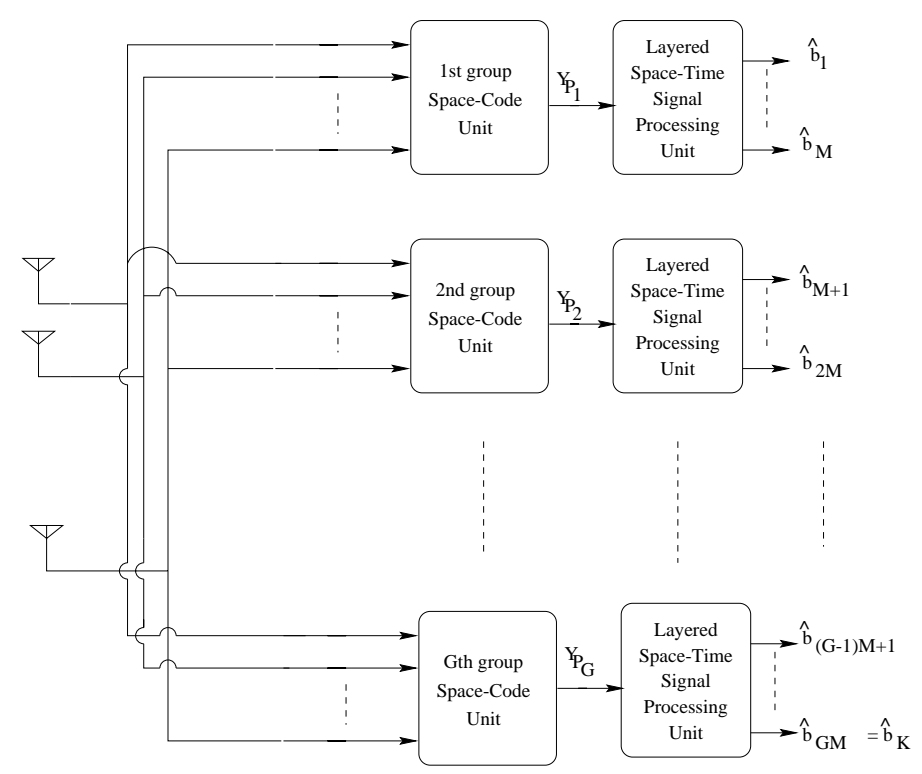

Figure 9: Parallel-Layered Space-Time Group Multiuser Detector

iteration $(i+1)$ is defined by

$$
\mathbf{Y}_{\mathbf{S}_{\mathbf{g}}}(i+1)=\sum_{p=1}^{P}\left[\mathbf{X}_{g_{p}}(i+1)\right]^{H} \mathbf{r}_{g_{p}}(i+1)
$$

where

$$
\mathbf{r}_{g_{p}}(i+1)=\mathbf{r}_{g_{p}}(i)-\left(\mathbf{X}_{p}\right)_{k_{i}} \hat{b}_{k_{i}} .
$$

and $\mathbf{X}_{g_{p}}(i+1)$ is obtained by expurgating the $k_{i}$ column of $\mathbf{X}_{g_{p}}(i)$. This iterative process is continued until all data has been retrieved.

\section{B. Parallel-Layered Space-time Group Multiuser Detector}

To further decrease the system design complexity, we propose here to retrieve the groups data sets in parallel. Under this assumption, no interference cancellation between groups is deployed in this case (See Fig. 9). This receiver type will be denoted by Parallel Group LAyered Space-Time MUltiuser Decorrelator or Parallel LASTG-MUD. It is clear that this new design leads to a faster receiver than the Serial LASTG-MUD since it needs only one group block timing to extract all the transmitted data from all the groups. However, the hardware requirement is multiplied by the number of existing groups. We should also mention that the performance of this system is expected to present some degradation compared to the previous one since each group sees an interference from all the other groups. The detection algorithm of the considered system is similar to the previous one (Sec. V. B) with some minor modifications, which consist of defining an estimated vector $\hat{\mathbf{b}}_{g}$ for each group instead of a common vector $\hat{\mathbf{b}}$ in the initialization part. The group input vectors to each group space-code unit are thus identical and equal to the received signals $\mathbf{r}_{p}$. 


\section{Complexity Computation}

Both Serial and Parallel LASTG-MUD detectors consist of two parts as described in Section V.A. and V.B. The first is a space/code matched filter, mainly used to separate the groups and cancel the group multi-access interference. The second part consists of a generalization of the layered space-time detection. The latter, assuming a zero-forcing criteria, computes a nulling vector for each user in order to estimate the transmitted data. In the proposed LASTG-MUD receivers, a user's signal is retrieved with regards to its group and independently of the users of the other groups. Therefore, the nulling vector of one user is chosen to be orthogonal to the space vector spanned only by the remaining undetected users in the same group. By doing so, Serial LASTG-MUD detection process, for example, involves the inversion of only the $G$ cross-correlation $M \times M$ matrices, $\tilde{\mathbf{R}}_{\mathbf{S}}^{(g)}$. In contrast, in LAST-MUD system, users signals are estimated regardless of their groups (See Section II), and the nulling vector of one user in this system is chosen to be orthogonal to all the remaining undetected users of all the groups. Hence, the inversion of the $K \times K$ space/code cross-correlation matrix is needed. A significant computational complexity reduction is therefore achieved with LASTG-MUD architectures.

Since the Serial and Parallel detectors have very similar structures, they involve the same number of arithmetic operations. Therefore, we compare only the complexities of LAST-MUD and Serial LASTGMUD. In order to estimate such a reduction, Table I computes the necessary arithmetic operations involved in retrieving all the transmitted symbols for both systems. We quantify in the following the complexity reduction as the complementary of the arithmetic-operations-number ratio of both receiver, that we denote

$$
1-\frac{\text { Serial LASTG-MUD }}{\text { LAST-MUD }}
$$

It is assumed that both systems use the same spreading factor $N$ and the number of receive antennas $P$. Numerical examples are also provided in Table I to emphasize the complexity reduction achieved with a disjoint group detection structure. For instance, when the system presents 12 users grouped in 2 groups of 6 users, almost $80 \%$ of complexity reduction is obtained, and $90 \%$ when users are grouped into 3 groups. Therefore, we notice that the complexity reduction increases with the number of groups in the system. However, in such a case, the performance in terms of bit error rate (BER) is not expected to improve for a fixed bandwidth, which involves a tradeoff between system complexity and BER performance.

\section{Performance Evaluation}

We investigate here the performance of Serial LASTG-MUD and Parallel LASTG-MUD using BPSK modulation. We are especially interested in the tradeoff between complexity reduction and bandwidth expansion in order to keep a fixed QOS. The assumptions made in Section V are considered in the following simulations. 
TABLE I

NUMBER OF ARITHMETIC OPERATIONS REQUIRED FOR THE DETECTION

\begin{tabular}{|c|c|c|c|c|}
\hline \hline & LAST-MUD & Serial LASTG-MUD & \multicolumn{2}{c|}{$\begin{array}{c}\text { Complexity Reduction } \\
\left(1-\frac{\text { Serial } L A S T G-M U D}{L A S T-M U D}\right)\end{array}$} \\
\cline { 3 - 5 } & $K$ Users, $P$ RX and Spr. fac $=N$ & $G$ groups with $M$ users, \\
& & $P$ RX and Spr. fac $=N$ & $\begin{array}{c}\mathrm{G}=2, \mathrm{M}=6, \\
\mathrm{P}=6, \mathrm{~N}=15\end{array}$ & $\begin{array}{c}\mathrm{G}=3, \mathrm{M}=4, \\
\mathrm{P}=6, \mathrm{~N}=15\end{array}$ \\
\hline \hline$(+)$ & $K^{4}+\frac{5}{3} K^{3}+(N+P) K^{2}$ & $G \times\left(M^{4}+\frac{5}{3} M^{3}+2(N+P+1) M^{2}\right.$ & $74.82 \%$ & $86.06 \%$ \\
& $+\left(N+P-\frac{5}{3}\right) K$ & $\left.+\left(N(G+1)+P-\frac{2}{3}\right) M\right)-N$ & \\
\hline$(-)$ & $K^{4}$ & $G \times\left(M^{4}-M^{2}+M N+N\right)$ & $86.83 \%$ & $95.44 \%$ \\
\hline$(\times)$ & $2 K^{4}+2 K^{3}$ & $G \times\left(2 M^{4}+2 M^{3}\right.$ & $80.44 \%$ & $89.88 \%$ \\
\hline$(/)$ & $+(N+2) K^{2}+K N$ & $\left.+N M^{2}+(N(G+2)+1) M\right)$ & & $87.181 \%$ \\
\hline$(\sqrt{ })$ & $2 K^{3}+2 K^{2}$ & $G \times\left(2 M^{3}+2 M^{2}\right)$ & $73.08 \%$ & $61.54 \%$ \\
\hline Total & $4 K^{4}+\frac{17}{3} K^{3}+\left(2 N+P+\frac{9}{2}\right) K^{2}$ & $G \times\left(4 M^{4}+\frac{17}{3} M^{3}+\left(4 N+2 P-\frac{1}{2}\right) M^{2}\right.$ & $80.31 \%$ & $90.43 \%$ \\
& $+\left(2 N+P-\frac{7}{6}\right) K$ & $\left.+\left(2 N(G+2)+P+\frac{5}{6}\right) M\right)$ & & \\
\hline \hline
\end{tabular}

\section{D.1. Serial LASTG-MUD}

In Figure 10, we evaluate the performance of the Serial LASTG-MUD, for 12 users divided into groups of $6,4,3$ and 2 users, with a variable spreading factor of 15, 32 and 64, respectively. From Appendix B, we know that the performance limit that we can achieve with $G$ groups of $M$ users is defined by the performance of LAST-MUD with the same configuration, under the necessary conditions, or by the performance of rT-BLAST decorrelator performance with $M$ transmit antennas. Hence, we plot in Figure 10 the performance of the rT-BLAST decorrelator with 2, 3, 4 and 6 transmit antennas for reference. A close observation of this figure and particularly to the system with 3 groups of 4 users, and a spreading factor $N=15$, indicates that at $7.5 \mathrm{~dB}$ SNR the BER performance is limited to $10^{-3}$ versus $10^{-6}$ with LAST-MUD under the same conditions. The complexity reduction is about $90 \%$ (See Table. I), but the performance also drops to about $10^{-3}$. Bandwidth expansion appears to be one solution. Thus, we consider $N=32$ and 64 in Figure 10. We notice that with $N=64$, we obtain a BER of the order of $10^{-5}$ at $7.5 \mathrm{~dB}$ SNR. The complexity reduction is defined in this case by

$$
1-\frac{\text { Serial } L A S T G-M U D(N=64)}{L A S T-M U D(N=15)}
$$

and is about $75 \%$.

\section{D.2. Parallel LASTG-MUD}

We analyze the performance of Parallel LASTG-MUD for 12 users divided also into 2, 3, ,4 and 6 groups, with $N=15,32$ and 64 , respectively. We found that the effect of grouping and spreading factor on the BER variations is almost the same as that of Serial LASTG-MUD (See Fig. 11). Some degradation in the performance is also noticed due to the absence of the interference cancellation between the groups. In fact, if we consider the example with $N=64$, we find that the BER is about $10^{-5}$ at 


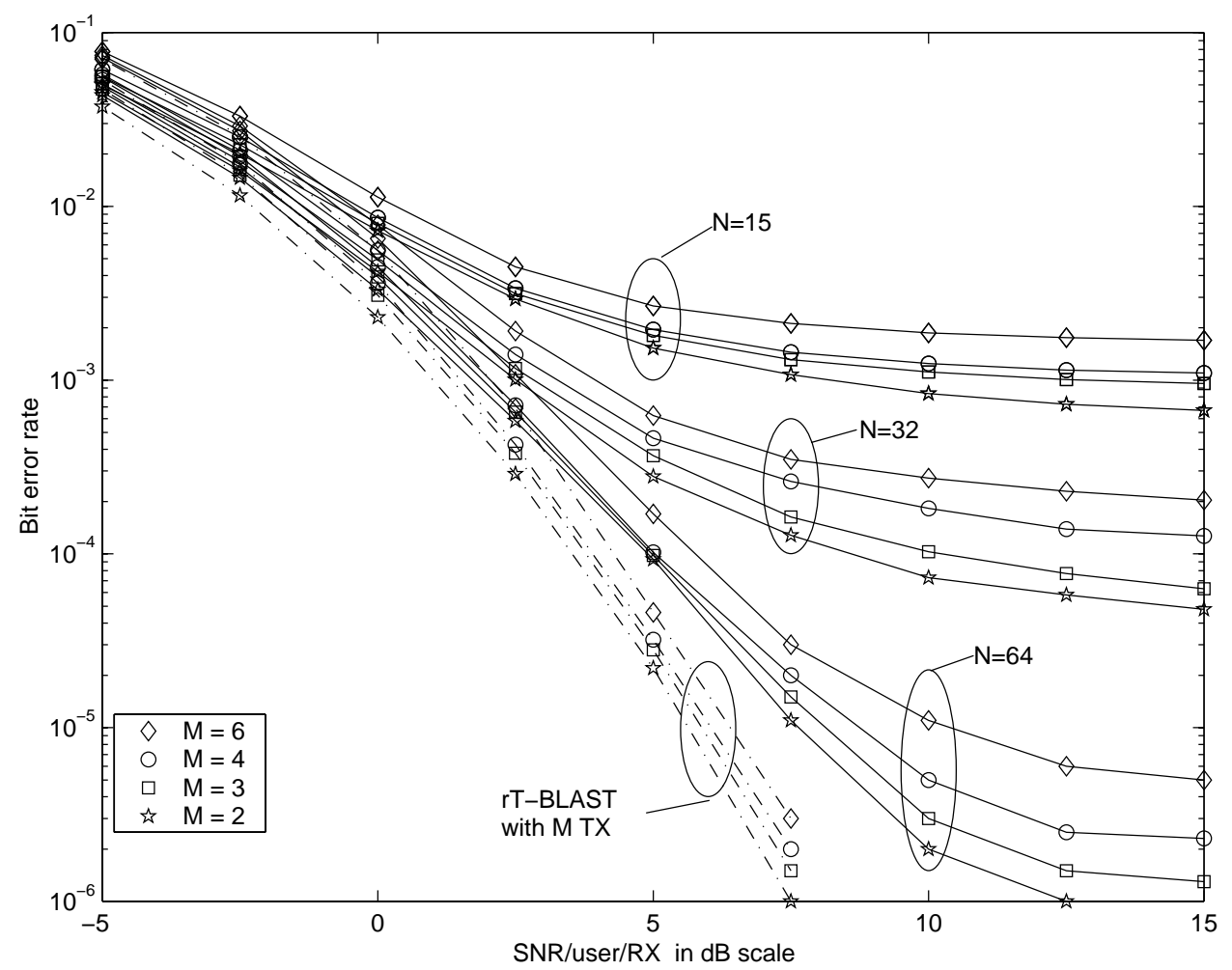

Figure 10: Serial LAyered Space-Time Group Multiuser Detector performance with BPSK, 12 users and 6 RX. The performance of $\mathbf{r T}$-BLAST detector is also shown.

10dB SNR with 2, 3, 4 and 6 groups. A complexity reduction of the order of $76 \%$ is also achieved in this case at the expense of bandwidth expansion by a factor $\frac{64}{15}$ and an SNR increase by $2.5 \mathrm{~dB}$ compared to LAST-MUD. However, the Parallel LASTG-MUD receiver converges much faster than the Serial LASTG-MUD receiver, since it needs only one group block timing to converge instead of $G$ block timing. Also note that the performance of these detectors could be further enhanced by considering more stages of interference cancellation.

\section{CONCLUSIONS}

In this paper, we apply layered space-time processing for multiuser detection communication systems in fading channels. The multiple transmit antennas in V-BLAST are treated as individual mobile station transmitters, while the base station consists of multiple receive antennas. In the proposed system, users are organized in groups and allocated a unique spreading code within the same group. Using these codes, we are able to separate the different groups, and layered space-time algorithm is then invoked to further remove the remaining interference between users.

We first applied the layered space-time algorithm for all the users independently of their groups (LAST-MUD). A significant performance improvement and increase in system capacity are obtained for very low spreading factors. In addition, we have considered the special case of real constellation based 


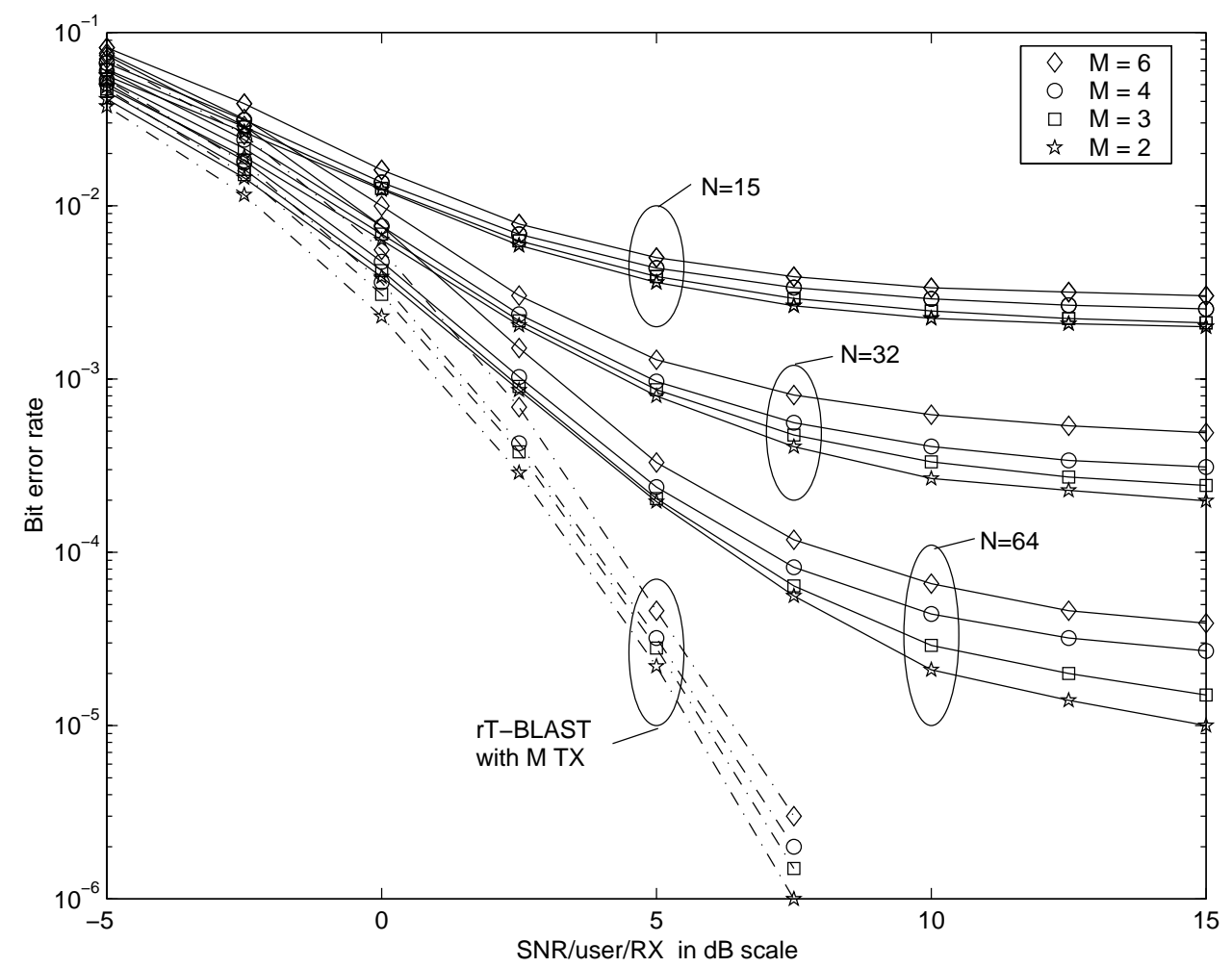

Figure 11: Parallel LAyered Space-Time Group Multiuser Detector performance with BPSK, 12 users and 6 RX. The performance of $\mathbf{r T - B L A S T}$ detector is also shown.

modulations. We derived two decorrelators based V-BLAST, rT and Tr-decorrelators and we discussed analytically and also by simulation their performance. We showed that the $\mathbf{r T}$-decorrelator outperforms the $\operatorname{Tr}$ receiver, and the gain in terms of BER or number of transmit antennas was very large. A Layered Space-Time Multiuser detector based on the $\mathbf{r T}$-decorrelator is then derived when real constellations are used. We also proposed reduced complexity receiver structures, which consist of applying the layered space-time detection algorithm disjointly at the group level in a serial and a parallel fashion. We denote these receivers Serial LASTG-MUD and Parallel LASTG-MUD, respectively. Finally, a tradeoff between system complexity, bandwidth expansion and BER performance is investigated.

\section{ACKNOWLEDGMENTS}

The authors wish to thank Min Yan from the Hong Kong University of Science and Technology for his assistance with the proof provided in Appendix B and his helpful comments.

\section{References}

[1] T. Ojanpera and R. Prasad, "An Overview of Third-Generation Wireless Personal Communications: A European Prospective," IEEE Personnel Communications, Vol. 5, No. 6, pp. 59-65, Dec. 1998. 
[2] A. Paulraj and C. Papadias, "space-time processing for Wireless Communications," IEEE Signal Processing Magazine, Vol. 14, No. 6, pp. 49-83, Nov. 1997.

[3] J. H. Winters, J. Salz, and R. D. Gitlin, "the impact of antenna diversity on the capacity of wireless communication systems," IEEE Transactions on Communications, Vol. 42, pp. 1740-1751, Feb./Mar./Apr. 1994.

[4] S. Verdú, "Multiuser Detection," Cambridge, MA: Cambridge University Press, Sept. 1998.

[5] L. C. Hui and K. B. Letaief, "Successive interference cancellation for multiuser asynchronous DS/CDMA detectors in multipath fading channels," IEEE Transactions on Communications, Vol. 46, No. 3, pp. 384-391, March 1998.

[6] W. K. Wong, R. D. Murch, and K. B. Letaief, "Optimizing Time and Space MIMO Antenna System for Frequency Selective Fading Channels," IEEE Journal on Selected Areas in Communications, Vol. 19, No. 7, July 2001.

[7] H. Huang, S. Schwartz, and S. Verdú, "Combined Multipath and Spatial Resolution for Multiuser Detection: Potentials and Problems," Proceedings of the IEEE International Symposium on Information Theory, pp. $380,1995$.

[8] M. Nagatsuka and R. Kohno, "A Spatially and Temporally optimal Multi-User Receiver using an Array antenna for DS/CDMA," IEICE Transactions on Communications, Vol. E78-B, No. 11, pp. 1489-1497, Nov. 1995.

[9] F. R. Farrokhi, G. J. Foschini, A. Lozano, and R. A. Valenzuela, "Link-optimal space-time processing with multiple transmit and receive antennas," IEEE Communications Letters, Vol. 5, Issue. 3, pp. 85-87, March 2001.

[10] C. Papadias and H. Huang, "Linear Space-Time multiuser Detection for Multipath CDMA Channels," IEEE Journal on Selected Areas in Communications, Vol. 19, No. 2,pp. 254-265, Feb. 2001.

[11] X. Wang and H. V. Poor, "Space-Time Multiuser Detection in Multipath CDMA Channels," IEEE Transactions on Signal Processing, Vol. 47, No. 9, pp. 2356-2374, Sep. 2001.

[12] G. J. Foschini and M. J. Gans, "On Limits of Wireless Communications in a Fading Environment When Using Multiple Antennas," Wireless Personal Communications, Vol. 6, No. 3, pp. 311-335, 1998.

[13] D. Chizhik, F. R. Farrokhi, J. Ling, and A. Lozano, "Effect of antenna separation on the capacity of BLAST in correlated channels," IEEE Communications Letters, Vol. 4 Issue. 11, pp. 337 -339, Nov. 2000.

[14] P. W. Wolniansky, G. J. Foschini, G. D. Golden, and R. A. Valenzuela, "V-BLAST: An Architecture for Realizing Very High Data Rates Over the Rich-Scattering Wireless Channel," Invited paper, Proc. ISSSE-98, Pisa, pp. 295-300, Italy, 1998. 
[15] G. J. Foschini, G. D. Golden, R. A. Valenzuela and P. W. Wolniansky, "Simplified processing for high spectral efficiency wireless communication employing multi-element arrays," IEEE Journal on Selected Areas in Communications, Vol. 17, No. 11, pp. 1841 -1852, Nov. 1999.

[16] V. Tarokh, N. Seshadri and A. R. Calderbank, "Space-time Codes for high data rate wireless communications: Performance criterion and code construction," IEEE Transactions on Information Theory, Vol. 44, pp. 744765, Mar. 1998.

[17] A. Lozano and C. Papadias, "layered Space-Time Receivers for Frequency -Selective Wireless channels," IEEE Transactions on Communications, Vol. 50, No. 1, pp. 65-73, Jan. 2002.

[18] N. Boubaker, K. B. Letaief, and R. D. Murch, "Performance of BLAST over frequency-selective wireless communication channels," IEEE Transactions on Communications, Vol. 50, No. 2, pp. 196-199, Feb. 2002.

[19] Xu Zhu and R. D. Murch, "MIMO-DFE based BLAST over frequency selective channels," IEEE Global Telecommunications Conference, GLOBECOM'01, Vol. 1, pp. 499-503, 2001.

[20] J. F. Frigon and B. Daneshrad, "Multiple input - multiple output (MIMO) receiver for wideband space-time communications," Proc. IEEE Int. Symp. on Personal, Indoor and Mobile Radio Communications, Vol. 1, pp. 164-168, Sept. 2001.

[21] A. Duel-Hallen, "Decorrelating decision-feedback multiuser detector for synchronous code-division multipleaccess channel," IEEE Transactions on Communications, Vol. 41, Issue. 2, pp. 285-290, Feb. 1993.

[22] G. Ginis and J. Cioffi, "On the Relation Between V-BLAST and the GDFE," IEEE Communications Letters, Vol. 5, No. 2, pp. 364-366, Sep. 2001.

[23] S. Kandala, E. S. Sousa, and S. Pasupathy, "Multi-user multi-sensor detectors for CDMA networks," IEEE Transactions on Communications, Vol. 43, No. 2(3), pp. 946-957, Feb.-March-April 1995.

[24] T. J. Lim, L. K. Rasmussen, and H. Sugimoto, "Relative Performance of BPSK and QPSK in the presence of Complex multiuser CDMA interference," Wireless Personal Communications, Vol. 13, No. 3, pp. 237-256, June 2000 .

[25] G. H. Golub and C. F. Van Loan, "Matrix Computations," Johns Hopkins University Press, Baltimore, MD, 1983.

[26] B. Noble and J. W. Daniel, "Applied Linear Algebra," Third edition, Prentice Hall, 1988.

[27] E. Bechenback and R. Bellman, "Inequalities," $2^{\text {nd }}$ revised printing, New York: Springer Verlag, pp. 69, 1965. 


\section{Appendix A}

In this appendix, we show by induction that the rT-BLAST decorrelator and the LAyered SpaceTime Multiuser Decorrelator (LAST-MUD) are equivalent when the network presents only one group of $M$ users and $P$ receive antennas. Thus, we consider rT-BLAST system with $M$ users, and a LASTMUD system with one group of $M$ users and a spreading factor $N$. We will first show that at the first iteration in the detection algorithm, both systems present the same cross-correlation matrix and equivalent received decision statistic vectors.

Consider the rT-BLAST receiver first. Using the notations defined in Section III, the space crosscorrelation matrix is given by

$$
\mathbf{R}_{\mathbf{r T}}=\operatorname{Re}\left(\mathbf{R}_{\mathbf{T r}}\right)=\operatorname{Re}\left(\mathbf{H}^{H} \mathbf{H}\right)=\operatorname{Re}\left[\begin{array}{cccc}
\sum_{p=1}^{P} h_{p 1}^{*} h_{p 1} & \sum_{p=1}^{P} h_{p 1}^{*} h_{p 2} & \cdots & \sum_{p=1}^{P} h_{p 1}^{*} h_{p M} \\
\sum_{p=1}^{P} h_{p 2}^{*} h_{p 1} & \sum_{p=1}^{P} h_{p 2}^{*} h_{p 2} & \cdots & \sum_{p=1}^{P} h_{p 2}^{*} h_{p M} \\
\vdots & \vdots & \ddots & \vdots \\
\sum_{p=1}^{P} h_{p M}^{*} h_{p 1} & \sum_{p=1}^{P} h_{p M}^{*} h_{p 2} & \cdots & \sum_{p=1}^{P} h_{p M}^{*} h_{p M}
\end{array}\right]
$$

The received decision statistic vector is an $M$ vector $\mathbf{Y}_{\mathbf{r T}}=\left[y_{r T_{1}}, \cdots, y_{r T_{M}}\right]^{T}$ where

$$
\mathbf{Y}_{\mathbf{r T}}=\operatorname{Re}\left[\begin{array}{c}
\sum_{k=1}^{M}\left(\sum_{p=1}^{P} h_{p 1}^{*} h_{p k}\right) b_{k}+\sum_{p=1}^{P} h_{p 1}^{*} n_{p} \\
\sum_{k=1}^{M}\left(\sum_{p=1}^{P} h_{p 2}^{*} h_{p k}\right) b_{k}+\sum_{p=1}^{P} h_{p 2}^{*} n_{p} \\
\vdots \\
\sum_{k=1}^{M}\left(\sum_{p=1}^{P} h_{p M}^{*} h_{p k}\right) b_{k}+\sum_{p=1}^{P} h_{p M}^{*} n_{p}
\end{array}\right]
$$

with the noise vector component having a covariance equal to $\sigma^{2} \mathbf{R}_{\operatorname{Tr}}$. In the LAST-MUD system, we have $G=1, K=M$ users and a spreading factor $N$. The spreading matrix will be given by

$$
\mathbf{S}=\left[\begin{array}{cccc}
s_{11} & s_{21} & \cdots & s_{M 1} \\
s_{12} & s_{22} & \cdots & s_{M 2} \\
\vdots & \vdots & \ddots & \vdots \\
s_{1 N} & s_{2 N} & \cdots & s_{M N}
\end{array}\right]=\left[\begin{array}{cccc}
s_{1} & s_{1} & \cdots & s_{1} \\
s_{2} & s_{2} & \cdots & s_{2} \\
\vdots & \vdots & \ddots & \vdots \\
s_{N} & s_{N} & \cdots & s_{N}
\end{array}\right]
$$

Since all the users belong to the same group, then the code cross-correlation matrix is given by

$$
\mathbf{S}^{T} \mathbf{S}=\left[\begin{array}{cccc}
\sum_{n=1}^{N} s_{1 n}^{2} & \sum_{n=1}^{N} s_{1 n} s_{2 n} & \cdots & \sum_{n=1}^{N} s_{1 n} s_{M n} \\
\sum_{n=1}^{N} s_{2 n}^{2} & \sum_{n=1}^{N} s_{2 n} s_{2 n} & \cdots & \sum_{n=1}^{N} s_{2 n} s_{M n} \\
\vdots & \vdots & \ddots & \vdots \\
\sum_{n=1}^{N} s_{M n}^{2} & \sum_{n=1}^{N} s_{M n} s_{2 n} & \cdots & \sum_{n=1}^{N} s_{M n} s_{M n}
\end{array}\right]=\left[\begin{array}{cccc}
1 & 1 & \cdots & 1 \\
1 & 1 & \cdots & 1 \\
\vdots & \vdots & \ddots & \vdots \\
1 & 1 & \cdots & 1
\end{array}\right]
$$

Define $\tilde{\mathbf{R}}_{p}=\mathbf{C}_{p}^{H} \mathbf{S}^{T} \mathbf{S} \mathbf{C}_{p}$, then

$$
\tilde{\mathbf{R}}_{p}=\left[\begin{array}{cccc}
h_{p 1}^{*} & 0 & \cdots & 0 \\
0 & h_{p 2}^{*} & \cdots & 0 \\
\vdots & \ddots & \ddots & \vdots \\
0 & \cdots & 0 & h_{p M}^{*}
\end{array}\right]\left[\begin{array}{cccc}
1 & 1 & \cdots & 1 \\
1 & 1 & \cdots & 1 \\
\vdots & \vdots & \ddots & \vdots \\
1 & 1 & \cdots & 1
\end{array}\right]\left[\begin{array}{cccc}
h_{p 1} & 0 & \cdots & 0 \\
0 & h_{p 2} & \cdots & 0 \\
\vdots & \ddots & \ddots & \vdots \\
0 & \cdots & 0 & h_{p M}
\end{array}\right]
$$


Thus,

Therefore,

$$
\tilde{\mathbf{R}}_{p}=\left[\begin{array}{cccc}
h_{p 1}^{*} h_{p 1} & h_{p 1}^{*} h_{p 2} & \cdots & h_{p 1}^{*} h_{p M} \\
h_{p 2}^{*} h_{p 1} & h_{p 2}^{*} h_{p 2} & \cdots & h_{p 2}^{*} h_{p M} \\
\vdots & \vdots & \ddots & \vdots \\
h_{p M}^{*} h_{p 1} & h_{p M}^{*} h_{p 2} & \cdots & h_{p M}^{*} h_{p M}
\end{array}\right]
$$

$$
\begin{aligned}
\tilde{\mathbf{R}}_{\mathbf{M U}} & =\operatorname{Re}\left(\sum_{p=1}^{P} \mathbf{R}_{p}\right) \\
& =\operatorname{Re}\left[\begin{array}{cccc}
\sum_{p=1}^{P} h_{p 1}^{*} h_{p 1} & \sum_{p=1}^{P} h_{p 1}^{*} h_{p 2} & \cdots & \sum_{p=1}^{P} h_{p 1}^{*} h_{p M} \\
\sum_{p=1}^{P} h_{p 2}^{*} h_{p 1} & \sum_{p=1}^{P} h_{p 2}^{*} h_{p 2} & \cdots & \sum_{p=1}^{P} h_{p 2}^{*} h_{p M} \\
\vdots & \vdots & \ddots & \vdots \\
\sum_{p=1}^{P} h_{p M}^{*} h_{p 1} & \sum_{p=1}^{P} h_{p M}^{*} h_{p 2} & \cdots & \sum_{p=1}^{P} h_{p M}^{*} h_{p M}
\end{array}\right] \\
& =\mathbf{R}_{\mathbf{r T}}
\end{aligned}
$$

The received statistic vector is an $M$ vector $\mathbf{Y}_{\mathbf{M U}}=\left[y_{M U_{1}} \cdots y_{M U_{M}}\right]^{T}$ defined by

$$
\mathbf{Y}_{\mathbf{M U}}=\operatorname{Re}\left[\begin{array}{c}
\sum_{k=1}^{M}\left(\sum_{p=1}^{P}\left(\sum_{n=1}^{N} s_{1 n} s_{k n}\right)\right. \\
\left.h_{p=1}^{*} h_{p k}\right) \\
\sum_{k=1}^{M}\left(\sum_{p=1}^{P}\left(\sum_{n=1}^{N} s_{2 n} s_{k n}\right)\right. \\
\left.h_{p 2}^{*} h_{p k}\right) \sum_{p=1}^{P} h_{p 1}^{*}\left(\sum_{n=1}^{N} s_{1 n} n_{p_{n}}\right) \\
\vdots \\
b_{k}+\sum_{p=1}^{P} h_{p 2}^{*}\left(\sum_{n=1}^{N} s_{2 n} n_{p_{n}}\right) \\
\sum_{k=1}^{M}\left(\sum_{p=1}^{P}\left(\sum_{n=1}^{N} s_{M n} s_{k n}\right) h_{p M}^{*} h_{p k}\right) b_{k}+\sum_{p=1}^{P} h_{p M}^{*}\left(\sum_{n=1}^{N} s_{M n} n_{p_{n}}\right)
\end{array}\right]
$$

Given that the spreading codes are the same for all the users, it follows that $\sum_{n=1}^{N} s_{i n} s_{j n}=1 \forall i, j \in$ $\{1, \ldots, M\}$ and

$$
\mathbf{Y}_{\mathbf{M U}}=\operatorname{Re}\left[\begin{array}{c}
\sum_{k=1}^{M}\left(\sum_{p=1}^{P} h_{p 1}^{*} h_{p k}\right) b_{k}+\sum_{p=1}^{P} h_{p 1}^{*}\left(\sum_{n=1}^{N} s_{1 n} n_{p_{n}}\right) \\
\sum_{k=1}^{M}\left(\sum_{p=1}^{P} h_{p 2}^{*} h_{p k}\right) b_{k}+\sum_{p=1}^{P} h_{p 2}^{*}\left(\sum_{n=1}^{N} s_{2 n} n_{p_{n}}\right) \\
\vdots \\
\sum_{k=1}^{M}\left(\sum_{p=1}^{P} h_{p M}^{*} h_{p k}\right) b_{k}+\sum_{p=1}^{P} h_{p M}^{*}\left(\sum_{n=1}^{N} s_{M n} n_{p_{n}}\right)
\end{array}\right]
$$

We note that the noise vector in $\mathbf{Y}_{\mathbf{M U}}$ is with a covariance $\sigma^{2} \mathbf{R}_{\mathbf{T r}}$, which is equal to $\sigma^{2} \tilde{\mathbf{R}}_{\mathbf{M U}}$. Hence, both received decision statistic vectors and cross-correlation matrices are equivalent in both systems at the first iteration. This implies that the first detected bit in both systems is the same.

Recall that the whole process includes $M$ steps. For the first step, we showed that both systems are equivalent. Now assume that they are equivalent in the $j$ th step and let us show that this is also true for the $(j+1)$ th step. Since equivalence in $j$ th step is assumed, then $\tilde{\mathbf{R}}_{\mathbf{M U}}(j)=\mathbf{R}_{\operatorname{Tr}}(j)$. Hence, their pseudo-inverse is the same and the order of the detected bits is the same in both systems based on Eqn. 4 . Thus, after interference cancellation, the system for the $(j+1)$ th step is equivalent to a deflated version of the $j$ th step from which users $k_{1}$ to $k_{j}$ have been removed. Therefore, $\tilde{\mathbf{R}}_{\mathbf{M U}}(j+1)=\mathbf{R}_{\operatorname{Tr}}(j+1)$. 
Then, based on Eqns. 7 and 8, the sufficient statistic vectors in the $(j+1)$ th step, $\mathbf{Y}_{\mathbf{M U}}(j+1)$ and $\mathbf{Y}_{\mathbf{r T}}(j+1)$ are equivalent. As a result, we conclude by induction that for any step $j \in\{1, \ldots, M\}$ both receivers, LAST-MUD and rT-BLAST, are equivalent when the system presents only one group of $M$ users. 


\section{Appendix B}

In what follows, $\mathbf{F}^{H}$ means the complex conjugate of $\mathbf{F}$ and $\mathbf{F}^{T}$ means the transpose of $\mathbf{F}$. Let $v_{1}, \cdots, v_{m}$ be $m$ complex $n \times 1$ vectors. $v_{1}, \cdots, v_{m}$ are said to be real-linearly independent [26] if and only if

$$
\forall \alpha_{1}, \cdots, \alpha_{m} \in R ; \alpha_{1} v_{1}+\cdots+\alpha_{m} v_{m}=0_{n \times 1} \Leftrightarrow \alpha_{1}=\cdots=\alpha_{m}=0
$$

Let $\mathbf{F}$ denote a $n \times m$ complex matrix, and $\mathbf{A}$ and $\mathbf{B}$ real $n \times m$ matrices, such that $\mathbf{F}=\mathbf{A}+i \mathbf{B}$. Assuming the columns of $\mathbf{F}$ are real-linearly independent, we would like to show the following proposition:

\section{Proposition 1:}

$$
\begin{cases}n \geq m & \Longleftrightarrow \mathbf{F}^{H} \mathbf{F} \text { is nonsingular } \\ (2 n) \geq m & \Longleftrightarrow \operatorname{Re}\left(\mathbf{F}^{H} \mathbf{F}\right) \text { is nonsingular }\end{cases}
$$

\section{Proof:}

We note that the first part of the proposition is given in [26]. However, for the second part, we would like to show that the real part of $\mathbf{F}^{H} \mathbf{F}$ is positive definite, which implies the invertibility of this real part in particular.

Let $\mathbf{J}$ denote the $(2 n) \times m$ matrix $\left[\begin{array}{l}\mathbf{A} \\ \mathbf{B}\end{array}\right]$. The real-linear independence of $\mathbf{F}$ means that the columns of the real matrix $\mathbf{J}$ are real-linearly independent. This implies that $2 n \geq m$.

Since the columns of $\mathbf{J}$ are linearly independent, we conclude that the matrix $\mathbf{J}^{T} \mathbf{J}$ which is equal to $\mathbf{A}^{T} \mathbf{A}+\mathbf{B}^{T} \mathbf{B}$ is positive definite. On the other hand, this is nothing but the real part of

$$
\mathbf{F}^{H} \mathbf{F}=\left(\mathbf{A}^{T}-i \mathbf{B}^{T}\right)(\mathbf{A}+i \mathbf{B})=\mathbf{A}^{T} \mathbf{A}+\mathbf{B}^{T} \mathbf{B}+i\left[\mathbf{A}^{T} \mathbf{B}+\mathbf{B}^{T} \mathbf{A}\right]
$$




\section{Appendix C}

Proposition 2 : $B E R_{\mathbf{r T}, k_{j}} \leq B E R_{\operatorname{Tr}, k_{j}}$.

\section{Proof:}

Under perfect interference cancellation assumptions, the obtained BERs by both receivers at the $j$ th iteration is reduced to

$$
\begin{aligned}
& B E R_{\mathbf{r} \mathbf{T}, k_{j}}=Q\left[\sqrt{\frac{\left|b_{k_{j}}\right|^{2}}{\sigma^{2}\left[\mathbf{R}_{\mathbf{r} \mathbf{T}}(j)\right]_{\left(k_{j}, k_{j}\right)}^{-1}}}\right] \\
& B E R_{\operatorname{Tr}, k_{j}}=Q\left[\sqrt{\frac{\left|b_{k_{j}}\right|^{2}}{\sigma^{2}\left[\mathbf{R}_{\operatorname{Tr}}(j)\right]_{\left(k_{j}, k_{j}\right)}^{-1}}}\right]
\end{aligned}
$$

From Eqn. 16, the $k_{j}$ user is defined by the minimum diagonal element of $\left[\mathbf{R}_{\operatorname{Tr}}(j)\right]^{-1}$. This implies that it is sufficient to show that

$$
\min _{l \in\{j, \ldots, M\}}\left[\mathbf{R}_{\mathbf{r} \mathbf{T}}(j)\right]_{\left(k_{l}, k_{l}\right)}^{-1} \leq \min _{l \in\{j, \ldots, M\}}\left[\mathbf{R}_{\mathbf{T r}}(j)\right]_{\left(k_{l}, k_{l}\right)}^{-1} .
$$

We begin by showing that the $k_{l}$ th diagonal element of $\left[\mathbf{R}_{\mathbf{r T}}(j)\right]^{-1}\left(=\left[\mathbf{R e}\left(\mathbf{R}_{\mathbf{T r}}(j)\right]^{-1}\right)\right.$ is greater than or equal to the $k_{l}$ th diagonal element of $\left[\mathbf{R}_{\operatorname{Tr}}(j)\right]^{-1}$, for any $l \in\{j \cdots M\}$. Note that since $\mathbf{R}_{\operatorname{Tr}}(j)$ is hermitian, its inverse is also hermitian and the diagonal elements of this inverse matrix are real.

Let $\mathbf{y}$ be a real vector and let $\mathbf{x}=\left[\operatorname{Re}\left(\mathbf{R}_{\operatorname{Tr}}(j)\right)\right]^{-1} \mathbf{y}$ (note that $\mathbf{x}$ is real). Then,

$$
\mathbf{x}^{T} \mathbf{R}_{\operatorname{Tr}}(j) \mathbf{x}=\mathbf{x}^{T} \operatorname{Re}\left(\mathbf{R}_{\operatorname{Tr}}(j)\right) \mathbf{x}=\mathbf{y}^{T}\left[R e\left(\mathbf{R}_{\operatorname{Tr}}(j)\right)\right]^{-1} \mathbf{y}=\mathbf{x}^{T} \mathbf{y} .
$$

Using a generalization of Bergstrom's inequality [27] which states that for any real $\mathbf{x}, \mathbf{y}$ and any positive definite matrix $\mathbf{A}$,

$$
\mathbf{x}^{T} \mathbf{A} \mathbf{x y} \mathbf{y}^{T}[\mathbf{A}]^{-1} \mathbf{y} \geq\left(\mathbf{x}^{T} \mathbf{y}\right)^{2}
$$

Then, it follows that

$$
\begin{aligned}
\mathbf{x}^{T}\left[\mathbf{R}_{\operatorname{Tr}}(j)\right] \mathbf{x y}^{T}\left[\mathbf{R}_{\operatorname{Tr}}(j)\right]^{-1} \mathbf{y} & \geq\left(\mathbf{x}^{T} \mathbf{y}\right)^{2} \\
\mathbf{y}^{T}\left[\operatorname{Re}\left(\mathbf{R}_{\operatorname{Tr}}(j)\right)\right]^{-1} \mathbf{y y}^{T}\left[\operatorname{Re}\left(\mathbf{R}_{\operatorname{Tr}}(j)\right)\right]^{-1} \mathbf{y} & \geq\left(\mathbf{y}^{T}\left[\operatorname{Re}\left(\mathbf{R}_{\operatorname{Tr}}(j)\right)\right]^{-1} \mathbf{y}\right)^{2} \\
\mathbf{y}^{T}\left[\mathbf{R}_{\operatorname{Tr}}(j)\right]^{-1} \mathbf{y} & \geq \mathbf{y}^{T}\left[\operatorname{Re}\left(\mathbf{R}_{\operatorname{Tr}}(j)\right)\right]^{-1} \mathbf{y}
\end{aligned}
$$

By choosing $\mathbf{y}$ to be the $k_{l}$ th unit vector, we get

$$
\begin{aligned}
{\left[\left(\operatorname{Re}\left(\mathbf{R}_{\operatorname{Tr}}(j)\right)\right)^{-1}\right]_{\left(k_{l}, k_{l}\right)} } & \leq\left[\left(\mathbf{R}_{\operatorname{Tr}}(j)\right)^{-1}\right]_{\left(k_{l}, k_{l}\right)}, \quad \forall l=j, \cdots, M \\
{\left[\left(\operatorname{Re}\left(\mathbf{R}_{\operatorname{Tr}}(j)\right)\right)^{-1}\right]_{(l, l)} } & \leq \min _{l \in\{1, \ldots, M\}}\left[\left(\mathbf{R}_{\operatorname{Tr}}(j)\right)^{-1}\right]_{\left(k_{l}, k_{l}\right)}, \quad \forall j=1, \cdots, M \\
\min _{l \in\{j, \ldots, M\}}\left[\left(\operatorname{Re}\left(\mathbf{R}_{\operatorname{Tr}}(j)\right)\right)^{-1}\right]_{\left(k_{l}, k_{l}\right)} & \leq \min _{l \in\{j, \ldots, M\}}\left[\left(\mathbf{R}_{\operatorname{Tr}}(j)\right)^{-1}\right]_{\left(k_{l}, k_{l}\right)}
\end{aligned}
$$

